\title{
MASSIVE: A Bayesian analysis of giant planet populations around low-mass stars
}

\author{
J. Lannier ${ }^{1}$, P. Delorme ${ }^{1}$, A. M. Lagrange ${ }^{1}$, S. Borgniet ${ }^{1}$, J. Rameau ${ }^{2}$, J. E. Schlieder ${ }^{3}$, J. Gagné ${ }^{4, \star}$, M. A. Bonavita ${ }^{5}$, \\ L. Malo ${ }^{6}$, G. Chauvin ${ }^{1}$, M. Bonnefoy ${ }^{1}$, and J. H. Girard ${ }^{7}$
}

1 Univ. Grenoble Alpes, Institut de Planétologie et d'Astrophysique de Grenoble (IPAG, UMR 5274), 38000 Grenoble, France e-mail: justine. lannier@univ-grenoble-alpes.fr

2 Institut de Recherche sur les Exoplanètes (iREx), Département de physique and Observatoire du Mont Mégantic, Université de Montréal, CP 6128, Succursale Centre-Ville, Montréal, QC H3C 3J7, Canada

3 NASA Ames Research Center, Space Science and Astrobiology Division, MS 245-6, Moffett Field, CA 94035, USA

${ }^{4}$ Department of Terrestrial Magnetism, Carnegie Institution of Washington, Washington, DC 20015, USA

5 Institute for Astronomy, The University of Edinburgh, Royal Observatory, Blackford Hill, Edinburgh, EH9 3HJ, UK

${ }^{6}$ CFHT Corporation, 65-1238 Mamalahoa Hwy, Kamuela, HI 96743, USA

7 European Southern Observatory, Alonso de Cordova 3107, Vitacura, Santiago, Chile

Received 2 February 2016 / Accepted 22 June 2016

\section{ABSTRACT}

\begin{abstract}
Context. Direct imaging has led to the discovery of several giant planet and brown dwarf companions. These imaged companions populate a mass, separation and age domain (mass $>1 M_{\text {Jup }}$, orbits $>5 \mathrm{AU}$, age $<1 \mathrm{Gyr}$ ) quite distinct from the one occupied by exoplanets discovered by the radial velocity or transit methods. This distinction could indicate that different formation mechanisms are at play.

Aims. We aim at investigating correlations between the host star's mass and the presence of wide-orbit giant planets, and at providing new observational constraints on planetary formation models.

Methods. We observed 58 young and nearby M-type dwarfs in $L^{\prime}$-band with the VLT/NaCo instrument and used angular differential imaging algorithms to optimize the sensitivity to planetary-mass companions and to derive the best detection limits. We estimate the probability of detecting a planet as a function of its mass and physical separation around each target. We conduct a Bayesian analysis to determine the frequency of substellar companions orbiting low-mass stars, using a homogenous sub-sample of 54 stars.

Results. We derive a frequency of $4.4_{-1.3}^{+3.2} \%$ for companions with masses in the range of $2-80 M_{\text {Jup }}$, and $2.3_{-0.7}^{+2.9} \%$ for planetary mass companions (2-14 $M_{\text {Jup }}$ ), at physical separations of 8 to $400 \mathrm{AU}$ for both cases. Comparing our results with a previous survey targeting more massive stars, we find evidence that substellar companions more massive than $1 M_{\text {Jup }}$ with a low mass ratio $Q$ with respect to their host star $(Q<1 \%)$, are less frequent around low-mass stars. This may represent observational evidence that the frequency of imaged wide-orbit substellar companions is correlated with stellar mass, corroborating theoretical expectations. Contrarily, we show statistical evidence that intermediate-mass ratio $(1 \%<Q<5 \%)$ companion with masses $>2 M_{\text {Jup }}$ might be independent from the mass of the host star.
\end{abstract}

Key words. planetary systems - stars: low-mass - methods: statistical - planets and satellites: formation

\section{Introduction}

After more than two decades of exoplanet discoveries (e.g., Wolszczan \& Frail 1992; Mayor \& Queloz 1995; Udalski et al. 2002), more than two thousand planets have been identified, mostly using radial velocity (hereafter RV) and transit methods. Both techniques have detected exoplanets with masses ranging from one Earth mass up to the brown dwarf mass regime ( 13-80 $M_{\text {Jup }}$ ), orbiting at close separations from their host star (usually within $5 \mathrm{AU}$ ). Direct imaging (DI) has provided data for about 60 large separation $(\geq 10 \mathrm{AU})$ massive planets and brown dwarfs for more than a decade ${ }^{1}$ (e.g., 51 Eri b, Macintosh et al. 2015; HD 95086b, Rameau et al. 2013a; GJ504b, Kuzuhara et al. 2013; 2MASS0103(AB)b, Delorme et al. 2013; $\beta$ Pictoris b, Lagrange et al. 2010; HR 8799bcde, Marois et al. 2008, 2010; 2M1207b, Chauvin et al. 2004). About a third of the imaged

\footnotetext{
^ Sagan Fellow.

1 http://exoplanet.eu/
}

substellar companions ( $\mathrm{SCs},<80 \mathrm{M}_{\text {Jup }}$ ) have young M-type dwarf host stars (Table 1).

DI is usually limited to the detection of distant young massive planets because of their more favorable planet-to-host-star contrast and their larger angular separation. Some of the limitations of DI can be mitigated by using thermal imaging (in $L^{\prime}$-band) where young massive planets emit most of their flux, and by taking advantage of the intrinsically lower flux of lowmass stars, which provides a more favorable star-planet contrast.

Both populations of planetary mass companions (hereafter PMCs, <14 $M_{\text {Jup }}$; close-in RV and transit PMCs on one hand, and wide-orbit DI PMCs on the other) challenge our view on planetary formation. Core accretion (CA, Pollack et al. 1996; Alibert et al. 2004) followed by planet migration is the preferred model to explain both the formation of solar system giant planets and hot Jupiters found around solar-type stars. Nevertheless, CA does not easily explain the massive gas giants imaged around low-mass stars, especially at large separations, even in the case of pebble accretion (Lambrechts \& Johansen 2012). 
Table 1. Giant planets and brown dwarfs imaged around self-luminous young M-dwarfs (source: http://exoplanet.eu).

\begin{tabular}{|c|c|c|c|c|c|}
\hline Name $^{\text {ref }}$ & SpT & $\begin{array}{c}d \\
(\mathrm{pc})\end{array}$ & $\begin{array}{l}\text { Age } \\
(\mathrm{Myr})\end{array}$ & $\begin{array}{c}\text { Comp. mass } \\
\left(M_{\mathrm{Jup}}\right)\end{array}$ & $\begin{array}{l}\text { Sep. } \\
(\mathrm{AU})\end{array}$ \\
\hline \multicolumn{6}{|l|}{ Planetary-mass companions } \\
\hline $2 \mathrm{M} \mathrm{0103(AB)} \mathrm{b}^{(1)}$ & M5,5 + M6 & $47.2 \pm 3.1$ & 30 & $13.0 \pm 1.0$ & 84.0 \\
\hline GU Psc $b^{(2)}$ & M3 & $48.0 \pm 5.0$ & $100 \pm 30$ & $11.0 \pm 2.0$ & $2000 \pm 200$ \\
\hline J0219-3925 B $\mathrm{B}^{(3)}$ & M6 & $39.4 \pm 2.6$ & $30-40$ & $12-15$ & $156 \pm 10$ \\
\hline FW Tau b $b^{(4)}$ & M4 & $145.0 \pm 15.0$ & $1.8_{-10}^{+0.2}$ & $10.0 \pm 4.0$ & $330 \pm 30$ \\
\hline DH Tau b $b^{(5)}$ & M0,5 & $\sim 140$ & $1.0 \pm 0.9$ & $11.0_{-0.003}^{+0.01}$ & 330 \\
\hline $2 \mathrm{M} 044144 \mathrm{~b}^{(6)}$ & M8,5 & 140.0 & 1.0 & $7.5 \pm 2.5$ & $15.0 \pm 0.6$ \\
\hline CHXR $73 b^{(7)}$ & M3,25 & $\sim 1.6$ & 2 & $12.0_{-5.0}^{+8.0}$ & 200 \\
\hline $2 \mathrm{M} 1207 \mathrm{~b}^{(8)}$ & M8 & $52.4 \pm 1.1$ & $8 \pm 3$ & $4.0_{-1.0}^{+6.0}$ & $46.0 \pm 5.0$ \\
\hline VHS 1256-1257 $\mathrm{B}^{(9)}$ & M7.5 & $12.7 \pm 1.0$ & $150-300$ & $11.2_{-15}^{+9.7}$ & $102 \pm 9$ \\
\hline Ross $458(\mathrm{AB}) \mathrm{c}^{(10)}$ & M2 & $114.0 \pm 2.0$ & $475 \pm 325$ & $8.5 \pm 2.5$ & 1168.0 \\
\hline ROXs 42B b $b^{(11)}$ & M0 & 135.0 & $2 \pm 1$ & $10.0 \pm 4.0$ & $140.0 \pm 10.0$ \\
\hline \multicolumn{6}{|l|}{ Brown dwarfs } \\
\hline 1RXS J034231.8+121622 B $\mathrm{B}^{(12)}$ & M4V & $23.9 \pm 1.1$ & $60-300$ & $35 \pm 8$ & $19.8 \pm 0.9$ \\
\hline FU Tau b $b^{(13)}$ & M7,25 & 140.0 & 1.0 & 15.0 & 800 \\
\hline CD-35 $2722 b^{(14)}$ & M1V & $21.3 \pm 1.4$ & $100 \pm 50$ & $31.0 \pm 8.0$ & $67.0 \pm 4.0$ \\
\hline GJ $3629 \mathrm{~B}^{(12)}$ & M3V & $22 \pm 3$ & $30_{-13}^{+30}$ & $46 \pm 6$ & $6.5 \pm 0.5$ \\
\hline 2MASS J15594729+4403595 B & M2 & $33 \pm 4$ & 120 & $43 \pm 9$ & $187 \pm 23$ \\
\hline UScoCTIO $108 b^{(15)}$ & M7 & $145.0 \pm 2.0$ & $11 \pm 2$ & $16.0_{-2.0}^{+3.0}$ & 670.0 \\
\hline GSC $6214-210 b^{(16)}$ & M1 & $145.0 \pm 14.0$ & $11 \pm 2$ & $17.0 \pm 3.0$ & 320.0 \\
\hline Oph $11 b^{(17)}$ & M9 & $145.0 \pm 20.0$ & $11 \pm 2$ & $21.0 \pm 3.0$ & $243 \pm 55.0$ \\
\hline 1RXS J235133.3+312720 $\mathrm{B}^{(12)}$ & M2 & $50 \pm 10$ & 120 & $32.0 \pm 6.0$ & $120 \pm 20$ \\
\hline
\end{tabular}

References. ${ }^{(1)}$ Delorme et al. (2013); ${ }^{(2)}$ Naud et al. (2014); ${ }^{(3)}$ Artigau et al. (2015); ${ }^{(4)}$ Kraus et al. (2014a); (5) Itoh et al. (2005); ${ }^{(6)}$ Todorov et al. (2010); ${ }^{(7)}$ Luhman et al. (2006); ${ }^{(8)}$ Chauvin et al. (2004); ${ }^{(9)}$ Gauza et al. (2015); ${ }^{(10)}$ Burgasser et al. (2010); ${ }^{(11)}$ Currie et al. (2014); (12) Bowler et al. (2015); ${ }^{(13)}$ Luhman et al. (2009); ${ }^{(14)}$ Wahhaj et al. (2011); ${ }^{(15)}$ Béjar et al. (2009); ${ }^{(16)}$ Ireland et al. (2011); (17) Allers et al. (2006).

Indeed, the mass of the planets are of the same order of magnitude (e.g., 2M0103b, Delorme et al. 2013) or even greater (2M1207b, Chauvin et al. 2004) than the total mass of the protoplanetary disc ( $\approx 10 \%$ of the star's mass). On the other hand, gravitational instability (GI, Boss 2011; Cameron 1978) provides an interesting alternative to $\mathrm{CA}$ to explain the formation of wide-orbit, massive planets within the disk of a low-mass star. Other initial conditions for the formation of PMCs, such as the position of the ice line and the stellar metallicity, impact their formation mechanisms. For instance, there are indications that planets with masses between $10 M_{\text {Earth }}$ and $4 M_{\text {Jup }}$ orbiting metal-poor stars are found at larger separations (Adibekyan et al. 2013).

Studying low-mass stars provides an opportunity to test the formation of PMCs at the low end of the stellar mass function. They also allow the study of a wider range of companion to star mass ratios. Different mechanisms might explain the formation of the planets, as a function of their mass ratio. In this paper, we will investigate whether there are different populations of planets orbiting low and high-mass stars.

From an observational point of view, Bonfils et al. (2013) found that the rate of close-in giant planets $(m \sin (i)=$ 100-1000 $\left.M_{\text {Earth }}\right)$ around M-type stars is low $(f<0.01$ for $P=1-10 d, f=0.02_{-0.01}^{+0.03}$ for $\left.P=10-100 d\right)$, while closein super-Earths are much more numerous $\left(f=0.35_{-0.11}^{+0.45}\right.$ for $P=10-100 d)$. Concerning higher-mass stars, Rameau et al. (2013b) find that between $10.8 \%$ and $24.8 \%$ of A to F type stars host at least one planet defined by the parameter intervals $[1,13] M_{\text {Jup }}$ and [1,1000] AU. Recently, Bowler et al. (2015) showed that few $\mathrm{M}$ dwarfs host giant planets closer than for $<1000 \mathrm{AU}$ and argue that there is no evidence of a relation between the wide-orbit giant planet ( $>10 \mathrm{AU}$ ) frequency and the stellar mass (for A, FGK and M stars). However, other studies combining different techniques of detection (RV and DI, micro lensing and RV) have shown that giant planets are less frequent around M dwarfs (respectively, Montet et al. 2014; Clanton \& Gaudi 2014).

In this paper, we present the results of the M-dwArf Statistical Survey for direct Imaging of massiVe Exoplanets (MASSIVE). Our sample is composed of 58 young and nearby M dwarfs observed in $L^{\prime}$-band for which we have follow-up data for all our candidate companions.

We present the MASSIVE survey in Sect. 2. Section 3 details our Bayesian approach to derive the frequency of planetary companions orbiting low-mass stars. In Sect. 4, we explore the influence of the stellar host mass on the planet occurrence by comparing the planetary companion frequency around low-mass stars in the MASSIVE survey and around a similar VLT/NaCo survey that targeted higher mass, A-F stars (Rameau et al. 2013b). We present our conclusions in Sect. 5. In all the following sections, we consider three types of companions: PMCs with masses $<14 M_{\text {Jup }}$, brown dwarfs with masses between 14 and $80 M_{\text {Jup }}$, and SCs that bring together PMCs and brown dwarfs ( $\left.<80 M_{\mathrm{Jup}}\right)$. 
Table 2. Observing periods. V stands for visitor mode, $\mathrm{S}$ for service mode.

\begin{tabular}{ccc}
\hline \hline Obs. period & Run & Mode \\
\hline Dec. 2009 & 084.C-0739 & S \\
Aug. 2012 & 089.C-0665(B) & V \\
Nov. 2012 and Feb. 2013 & 090.C-0698(A) & V \\
Nov. 2012 & 090.C-0728(A) & S \\
Aug. 2013 & 291.C-5031(A) & S \\
\hline
\end{tabular}

\section{The MASSIVE survey}

\subsection{Observations}

\subsubsection{Sample selection}

58 M-dwarfs have been observed. This sample was built as follows:

- Age: we selected our targets from the known members of young stellar associations (e.g., Zuckerman et al. 2004). We selected 10 stars from TW Hydrae (5-15 Myr; we use 8 Myr, hereafter TWA, Kastner et al. 1997, Weinberger et al. 2013, and Webb \& Zuckerman 1999), 22 stars from the $\beta$ Pictoris moving group (20-26 Myr; we use $21 \mathrm{Myr}$, hereafter BPMG, Binks \& Jeffries 2014; Malo et al. 2014b; Mamajek \& Bell 2014), 8 stars from Tucana-Horologium (20-40 Myr; we use 30 Myr, hereafter THA, Zuckerman \& Webb 2000; Kraus et al. 2014b), 3 stars from Columba (20-40 Myr; we use 30 Myr, hereafter COL, Torres et al. 2008), 1 star from Argus (30-50 Myr; we use $40 \mathrm{Myr}$, Makarov \& Urban 2000), and 12 stars from ABDoradus (110-130 Myr; we use $120 \mathrm{Myr}$, hereafter ABDor, Luhman et al. 2005; Barenfeld et al. 2013). Two other targets are young, but do not belong a stellar association.

The membership of our targets is based on publications listed in Table 3. The number of known young, M-type dwarfs has increased substantially thanks to recent surveys (such as 2MASS, ROSAT, Galex, and WISE) and to efforts to identify and characterize new, low-mass members of young moving groups (e.g., Torres et al. 2008; Schlieder et al. 2012; Malo et al. 2013; and Gagné et al. 2015b).

- Distance: the M dwarfs in our sample are nearby $(d<62 \mathrm{pc})$ so that we can probe small star-companion projected separations, typically down to 5-10 AU.

- Brightness: the sample stars are brighter than $K_{\mathrm{S}}=12$ to allow for good AO correction and for the detection of lowmass planets.

These criteria are summarized for each target in Table 3. The closest and the youngest stars were observed in higher priority.

\subsubsection{The data}

Data were acquired from December 2009 to February 2013, over five runs (see Table 2) with VLT/NaCo (Rousset et al. 2003). The observing conditions are described in Table D.8. NaCo includes an adaptive optics system (NAOS) and a near-infrared camera (CONICA, Lenzen et al. 2003). It was mounted at the Nasmyth focus of the $8.2 \mathrm{~m}$ telescope UT4 at the time of the observations. CONICA is equipped with a CCD infrared camera, and an Aladdin 3 detector $(1024 \times 1024$ pixels $)$. We used the available infrared wavefront sensor (IRWFS/NAOS) to close the adaptive optics (AO) loop on faint red stars such as most MASSIVE targets.
We used the L27 camera (27.1 mas/pixel, and a field of view of $\left.28^{\prime \prime} \times 28^{\prime \prime}\right)$ and the cube mode of $\mathrm{NaCo}$, recording data-cubes of single frames with exposure times as low as $0.175 \mathrm{~s}$. We observed in $L^{\prime}$ band $(\lambda=3.8 \mu \mathrm{m}, \Delta \lambda=0.62 \mu \mathrm{m})$ to improve our sensitivity to the coolest and therefore lowest-mass companions. We used the pupil tracking mode with a high parallactic angle in order to apply Angular Differential Imaging (ADI, Lafrenière et al. 2007; Marois et al. 2006), a method that allows speckle subtraction and deeper close-in sensitivity. Otherwise, we used regular field tracking.

\subsection{Data reduction}

We use our Interactive Data Language (IDL) pipeline developed for $\mathrm{AO}$ reduction at the Institut de Planétologie et d'Astrophysique de Grenoble (IPAG) to reduce the VLT/NaCo data. The reduction steps are described in Lagrange et al. (2010) and Delorme et al. (2012). They include bad pixel removal, flatfielding, and frame recentering. Two different methods are used to detect faint companions in the data, dependending on the separation from the star.

At separations closer typically than $0.5^{\prime \prime}$, noise is dominated by quasi-static speckles. To mitigate this and reveal faint, closein companions, we employ a modified angular differential imaging (ADI) algorithm called Smart ADI (SADI, Marois et al. 2006). The reference point spread function used for PSF subtraction in the SADI framework were derived from a restricted number of images (10) where each had rotated by at least one Full width at half maximum (FWHM) at a separation of $0.4^{\prime \prime}$. Candidate companions in this region are identified by visual inspection of the residual images.

At separations typically larger than $0.5^{\prime \prime}$, where the noise in $L^{\prime}$-band is background limited, we apply a median-filtering technique. For each pixel in the region of interest, we subtract the median in a $20 \times 20$ pixel box centered on that pixel. This step is performed on each frame before stacking the images. Candidate companions are identified in this region using the automatic point source detection routine described in Delorme et al. (2012). When the field rotation is too small to apply SADI, which is the case for 18 of our targets, we only apply median-filtering.

By combining these complementary approaches to candidate detection, we maximize our sensitivity at all separations. The boundary of the inner/outer zone that are used for each target are given in Table D.9 and detailed in Sect. 2.4.2.

\subsection{Detection results}

\subsubsection{Companion detections}

We detail hereafter the status of two companions already reported in previous papers and that are part of MASSIVE: 2MASS J01033563-5515561(AB)b and 2MASS J12073346$3932539 \mathrm{~b}$. In addition, we present the peculiar case of 2MASS J08540240-3051366, an M-type star that we excluded from MASSIVE.

\section{MASS J01033563-5515561(AB)b}

2MASS J01033563-5515561(AB), hereafter 2MASS0103(AB), is a $30 \mathrm{Myr}$ M-dwarf binary located at $47 \mathrm{pc}$. Delorme et al. (2013) discovered an exoplanet orbiting this target, with an estimated mass of 12-14 $M_{\text {Jup }}$ using BT-Settl models 


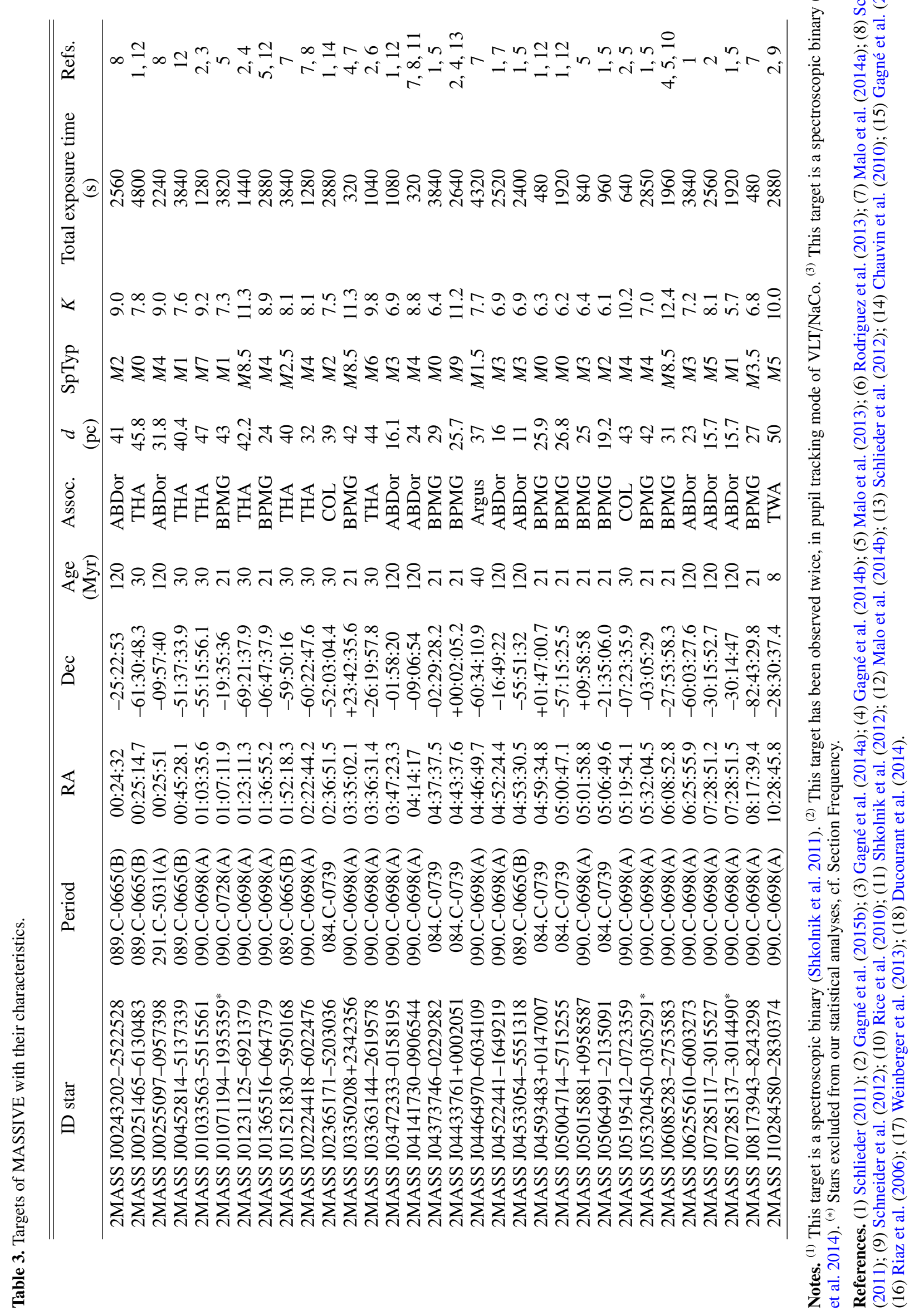


J. Lannier et al.: MASSIVE: A Bayesian analysis of giant planet populations around low-mass stars

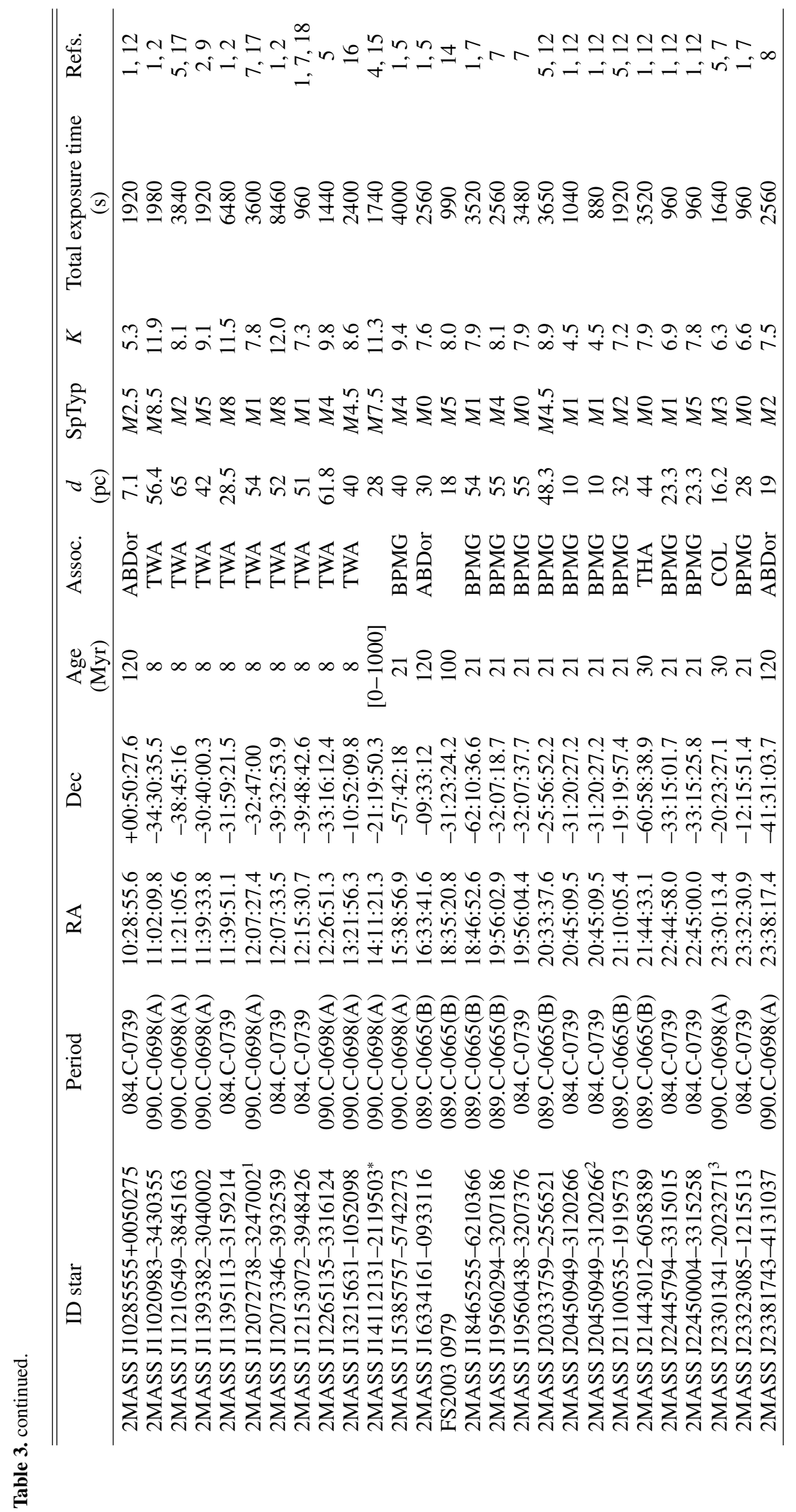


(Allard et al. 2012). We do not present any new result with respect to this target.

\section{MASS J12073346-3932539b}

2MASS J12073346-3932539 (hereafter 2M1207) is an $8 \mathrm{Myr}$ brown dwarf located at 52.4 pc. Chauvin et al. (2005) reported the discovery of an exoplanet orbiting this target.

$2 \mathrm{M} 1207 \mathrm{~b}\left(4 \pm 1 M_{\mathrm{Jup}}\right)$ is at a separation of $772 \pm 4$ mas $\left(\mathrm{PA}: 125.4 \pm 0.3^{\circ}\right), 768 \pm 5$ mas (PA: $125.4 \pm 0.3^{\circ}$ ), and $776 \pm 8$ mas $\left(\mathrm{PA}: 125.5 \pm 0.3^{\circ}\right)$ from its host star, respectively, for the VLT/NaCo data from 27/04/2004, 05/02/2005, and 31/03/2005. In the 2009 data, we find a separation of $756 \pm 12$ mas, with a position angle of $124.6^{\circ}$. Our new measurement thus overlaps with the previously reported results and no additional contraints on the orbit is added.

\section{MASS J08540240-3051366B}

We conducted an in-depth analysis (Appendix C) of 2MASS J08540240-3051366 (hereafter 2MASS J0854) because we identified a potentially interesting bound companion around it. However, a deeper analysis, using a new radial velocity measurement, revealed that the system is likely older than suspected (several Gyr instead of $10 \mathrm{Myr}$ ) and that the companion is a low-mass star. Thus, we do not include this target in our analysis.

\subsubsection{Background stars}

Nine MASSIVE targets were identified with candidate companions, in addition to 2MASS0103(AB) and 2M1207. Two methods were used to discriminate background objects from bonafide companions:

1. We used data obtained at different epochs, to test whether or not the candidate and the star shared the same proper motion. If they do, this would mean that they are gravitationally bound.

2. We used data obtained at different wavelengths when it was possible ( $J, H, K_{\mathrm{s}}, L^{\prime}$-bands), to compare the colors of the candidate with that expected for a background contaminant or a young planet companion. Indeed, young low gravity companions have much redder colors than main sequence contaminants: for instance, at $20 \mathrm{Myr}$, the BT-Settl models predict that a $5 M_{\text {Jup }}$ planet would have $H-L^{\prime} \approx 2.5$, while one of the reddest contaminants such as a 5 Gyr $0.3 M_{\odot}$ star, would have $H-L^{\prime} \approx 0.6$.

We find that all of them are background stars. The targets with background stars are: 2MASS J06255610-6003273, 2MASS J11210549-3845163, 2MASS J18465255-6210366, 2MASS J01365516-0647379, 2MASS J05015881+0958587, 2MASS J04522441-1649219, 2MASS J07285117-3015527, 2MASS J12073346-3932539, 2MASS J15385757-5742273 (see Fig. D.5).

\subsubsection{Visual binary stars}

We identified 13 close binary $\left(<1^{\prime \prime}\right)$ stars, which are reported in Table D.1. Some of them are very well resolved while others are tight or suspected binaries (some data show elongated intensity distributions). These binaries are studied within a larger sample of young binaries in another paper (Bonavita et al. 2016).

\subsection{Detection limits in mass and semi-major axis for MASSIVE}

In this section, we present the performances of MASSIVE in a (mass, semi-major axis) parameter space.

\subsection{1. $2 \mathrm{D}$ contrast maps}

Since we apply a different reduction technique at close and at large separations to detect SCs (see Sect. 2.2), we also derive 2D detection contrast maps in each case (see Delorme et al. 2012). Since the focus of this article is a statistical analysis, we choose to present the results from the simple and robust ADI technique such as SADI, rather than the slightly more sensitive LOCI (locally optimized combination of images, Lafrenière et al. 2007) or PCA (principal component analysis, Soummer et al. 2012) analyses that use subjective parameters (such as the size of the optimization zones in LOCI or the numbers of components kept in PCA) that can be optimized for individual analyses but not easily adapted for such a systematic analysis. The benefit of LOCI or PCA in sensitivity are moderated in $L^{\prime}$-band because the speckle-dominated area is smaller. We therefore caution that our detection limits on individual stars are probably conservative at short separations (see Table D.9) by typically a few tenths of magnitudes with respect to the best performance that could be achieved with a carefully tuned LOCI or PCA analysis.

\subsection{2. $2 \mathrm{D}$ mass detection limit maps}

We use Baraffe et al. (2003) evolution models that assume a hot start together with BT-Settl model atmosphere (Allard et al. 2012) to estimate the masses of planets detectable at $5 \sigma$ given the computed contrast limits. We emphasize that the systematic errors of the models are very difficult to quantify, and can be significant, see for example Dupuy et al. (2014). However, hot-start models have been commonly used for a long time and provide the best reference for the comparison between various imaging surveys.

Since the inner and outer regions of the 40 stars where SADI was applied are dominated by different sources of noise, we generate composite maps to cover the full range of possible semimajor axes. We determine the location of the boundary between the inner and outer regions on a star by star basis by comparing the detection limits reached using each image processing technique (the brighter the target, the wider the boundary, see Table D.9).

Figure 1 gives the median detection performances of MASSIVE in terms of contrasts in $L^{\prime}$-band and mass as a function of semi-major axis. The median detection performances for MASSIVE in apparent magnitudes of companions, is $L^{\prime} \approx$ 17 mag at $1^{\prime \prime}$ (at $5 \sigma$ ) and further away. The contrasts reachable at $L^{\prime}$ are presented in Table D.9. The MASSIVE survey is the deepest DI survey yet conducted on M dwarfs.

\subsubsection{Detection probabilities}

Using the 2D mass detection limit maps that we built in Sect. 2.4.2, we estimate the probability to detect companions orbiting our stars, as a function of given mass and semi-major axis.

We use an optimized version (Rameau et al. 2013b) of the original MESS code (Bonavita et al. 2012) which takes into account projection effects that can hide a companion behind its host star on a fraction of its orbits. Hence the detection limits 


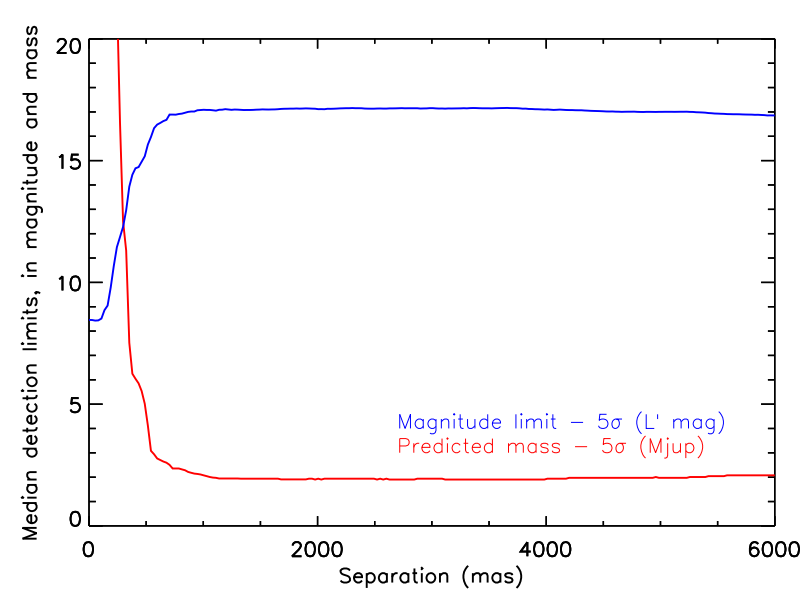

Fig. 1. Median detection performances for MASSIVE in magnitude ( $L^{\prime}$-band) and mass $\left(M_{\text {Jup }}\right)$.

represent the sensitivity to planets according to their semi-major axis (hereafter, SMA) and not to their projected separations. From a technic point of view, MESS is a Monte Carlo simulation code that builds synthetic planet populations described in terms of frequency, orbital elements and physical properties. It uses a semi-empirical approach to generate the planet populations using a regularly spaced grid. We generate 100000 planets for each [mass,SMA] pair of the grid. The semi-major axis range is 0.5 to $1000 \mathrm{AU}$ with steps of $2 \mathrm{AU}$ and the masses are in the range $[0.5,80] M_{\text {Jup }}$ with steps of $0.16 M_{\text {Jup }}$. The MESS code computes the SMA for each synthetic planet for a given mass and period. It also assigns eccentricities, inclinations, longitudes of periastron, longitudes of ascending node and times of periastron passage for each orbit, assuming uniform distributions. The eccentricity values marginally affect the detection probabilities, while the inclination strongly affects the detection probabilities.

We apply the MESS code to all of our targets, taking into account the host star age, distance, and mass (using BT-Settl models), as well as the masses and semi-major axes of the simulated planets, to derive mean planet detection probabilities for all MASSIVE targets (see Fig. 2).

We found that MASSIVE has a good sensitivity (mean detection probability $>50 \%$ ) in the range 8 to $400 \mathrm{AU}$ and 2 to $80 M_{\text {Jup }}$ (see Fig. 2) from the generation of 100000 planets for each point of the [mass,SMA] grid. We consider only this best parameter range in most of the following analysis.

\section{Frequency of planetary companions orbiting low-mass stars of the MASSIVE survey}

We detail the statistical formalism used for our Bayesian analysis in Appendix A.

For this analysis (Sects. 3 and 4), we exclude 4 targets out of the 58 targets of the MASSIVE sample, either because the estimated age is too uncertain (i.e., not members of young moving groups, like it is the case for 2MASS J14112131-2119503), or because of the close binarity of some targets that makes the total mass too big to consider it as a low-mass system (this is the case for 2MASS J01071194-1935359, 2MASS J053204500305291 and 2MASS J07285137-3014490, see Table D.2). However, we kept in our survey components of wide-separation binaries for which our AO observations allowed to look for SCs around each component individually. This led to a homogeneous sample of 54 targets.

\subsection{Optimal parameter range to be considered in our formalism}

The choice of parameter range on which we carry out our statistical analysis has a direct impact on the results. For instance, if we choose a broad range, such as [1-13] $M_{\text {Jup }}$ and [1-1000] AU used by Rameau et al. (2013b), we include probability integration ranges like [1-8] AU or [400-1000] AU where our probability of detecting any planet is close to zero. This would lead to larger errors because of the lack of actual information injected into the probability calculation. Also, since we carried out a Bayesian analysis, including such ranges with no observational constraints would artificially bias the results toward a posterior that is close to the supposed prior. We therefore choose to focus on ranges of mass and SMAs for which the MASSIVE observational constraints are stronger (cf. Sect. 2.4.3), that is [2, 80] $M_{\text {Jup }}$ or $[1,80] M_{\text {Jup }}$ at $[8,400]$ AU.

\subsection{Results of our Bayesian analysis}

\subsubsection{Working hypotheses}

We choose to address several distinct statistical questions (hereafter, we use $Q$ as the mass ratio between a host star and its planet):

- What is the probability to have a massive companion ( $2 M_{\text {Jup }}<M<80 M_{\text {Jup }}$ ) regardless of its $Q$ value? (case 1 )

- What is the probability to have a massive companion ( $2 M_{\text {Jup }}<M<80 M_{\text {Jup }}$ ) at an intermediate mass ratio $0.01 \leq Q \leq 0.05$ ? (case 2)

- What is the probability to have a massive companion ( $1 M_{\text {Jup }}<M<80 M_{\text {Jup }}$ ) at a low mass ratio $Q \leq 0.01$ ? (case 3)

We choose an upper limit of 0.05 for the intermediate mass ratio (case 2), because it excludes most low-mass star companions. Such companions might make the system appear as a binary, which could cause a selection bias because known binaries were excluded in the survey of Rameau et al. (2013b). Choosing such an upper limit in mass ratio keeps only the very high contrast companions in the selection range, and therefore effectively removes this possible bias.

We choose $1 M_{\text {Jup }}$ as a lower mass limit for case 3 since our previous lower mass limit ( $2 M_{\mathrm{Jup}}$, cases 1 and 2 ) is not relevant for such low $Q$ because in practice it excludes all the lowest mass stars $\left(<0.2 M_{\odot}\right)$ of our sample. Indeed, by choosing $1 M_{\text {Jup }}$ instead of $2 M_{\text {Jup }}$, we increase the effective number of LMSs (number of stars in sample times average companion detection probability in the chosen semi-major axis and mass range) by $15 \%$ (see Table D.3). So, decreasing the lower-mass limit allows to better constrain case 3 , which is not true for cases 1 and 2. Our statistical formalism correctly addresses the fact that the sensitivity of MASSIVE concerning some stars for case 2 and 3 is very weak or even zero, with the hypothesis that the planetary frequency in the considered mass range is homogeneous.

More precisely, our three cases correspond to 3 working hypotheses:

Case 1. We consider all detections, regardless of their $Q$ : we want to know the frequency of stars hosting substellar companions (PMCs and brown dwarfs). We will therefore consider all known detections, namely the systems 2MASS0103(AB) and $2 \mathrm{M} 1207$.

Case 2. We want to know the frequency of stars hosting SCs having a moderate mass ratio $Q$ (that is $0.01<Q<0.05$ ) 

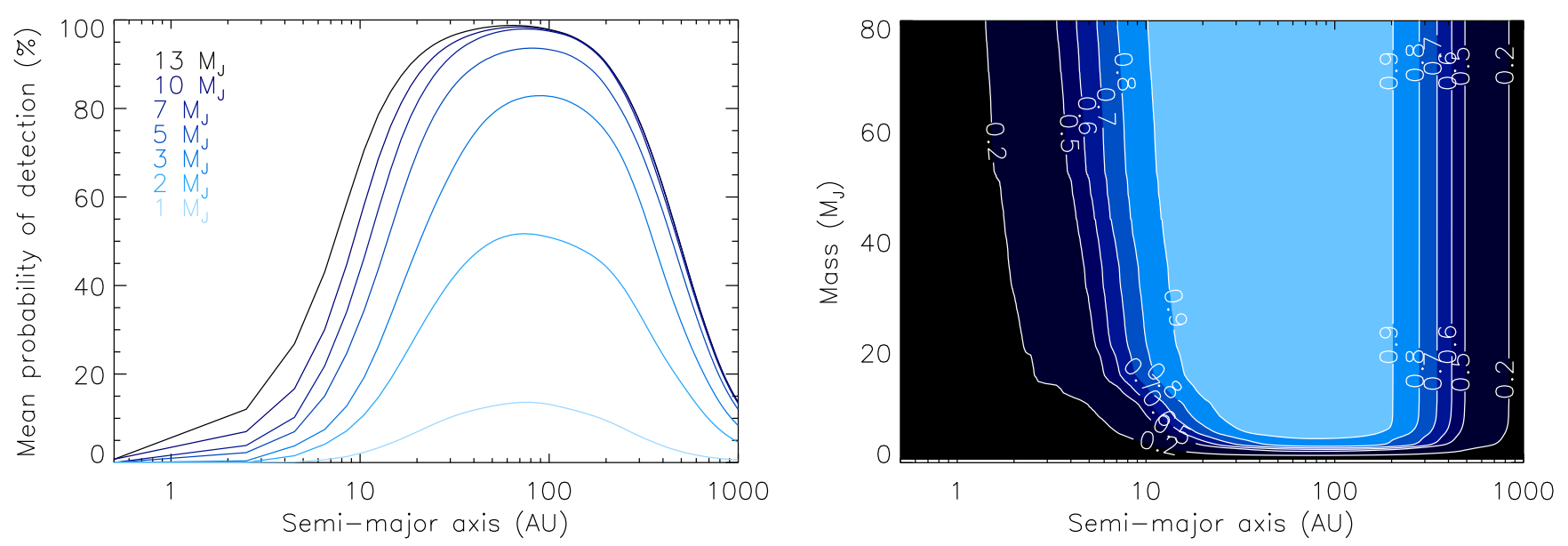

Fig. 2. MASSIVE survey mean detection probability curves (left) and contour plot of detection probabilities (right) for several masses, as a function of semi-major axis.

only. With the limitations detailed below (see Sect. 3.2.2), this intermediate $Q$ range would tentatively target objects formed via GI. For this second case, there is one detection in our sample (2MASS0103(AB)b). The median value for the MASSIVE targets is $0.45 M_{\odot}$, corresponding to $22.5 M_{\text {Jup }}$ and $4.5 M_{\text {Jup }}$ companion upper mass limit for $Q=0.05$ and $Q=0.01$ respectively. Case 3. We consider SCs with a very low mass ratio $Q, Q \leq 0.01$. With the limitations detailed below (see Sect. 3.2.2), this very low $Q$ range tentatively targets objects that more likely formed via $\mathrm{CA}$. There are no detections of such low- $Q$ companions in the MASSIVE survey.

\subsubsection{Companion frequencies for low-mass stars in MASSIVE}

We use Eqs. (4) and (5) to determine the minimum and maximum value of the substellar companion frequency given a confidence level, respectively $f_{\min }$ and $f_{\max }$, using Bayes rule and a binomial likelihood distribution (see Appendix A). In the case where no companion is detected in a given parameter range, we use Eq. (9) to determine $f_{\max }$. We use both natural conjugate priors and uniform priors. Using a uniform prior is a strong hypothesis in terms of planet frequency probability distribution, and a hypothesis that is rather discrepant with the relatively low observed giant planet frequency. We nevertheless use it to compare our results with published ones which generally use it, mostly because uniform prior does not use any observational information, in other words it is fixed regardless of the data. Note also that the flat prior is not an uninformative prior. We also use our observational constraints by estimating a natural conjugate prior (see Appendix A) in our empirical Bayesian analysis. We consider that this conjugate prior is better adapted to our analysis than the uniform prior because it is much more consistent with observations.

Our statistical results concerning the MASSIVE survey are reported in Table D.6, Figs. D.1 and D.2. The figures show the frequency distributions derived considering the whole mass and SMA ranges, ie $[0.5,80] M_{\text {Jup }}$ and $[0.5,1000]$ AU. These parameter ranges are not optimal for our analysis (cf. Sect. 3.1) but present the advantages to show how sensitive the frequency distributions are with respect to the parameters choice. The results that we report in the next sections are based on the use of optimized parameters ranges (cf. Sect. 3.1). We find that $2.3_{-0.7}^{+2.9} \%$
(68\% CL) of our low-mass stars host at least one giant planet in the mass range 2-14 $M_{\text {Jup }}$ and SMA range 8-400 AU. To derive this value, we use the natural conjugate prior and took into account the detection of the 2MASS0103 system. When we consider the same SMA range and a broader mass range, that is $2-80 M_{\text {Jup }}$, we find that $4.4_{-1.3}^{+3.2} \%$ of our low-mass stars host at least one SC (68\% CL, case 1), using a natural conjugate prior and considering the detection of 2MASS0103(AB)b and $2 \mathrm{M} 1207 \mathrm{~b}$. The frequency distributions are wider when considering the uniform prior instead of the conjugate prior (see Table D.6, Figs. D.1 and D.2): the lower value does not change significantly, which is due to the fact that uniform priors assume more numerous planets, while conjugate priors use the observations that revealed few companions.

Our frequencies are lower for cases 2 and 3 which is expected because cases 2 and 3 companions are subsamples of case 1 companions. We derive that $2.5_{-0.8}^{+3.1} \%$ of the low-mass stars host at least one SC $\left(68 \% \mathrm{CL}\right.$, case $2: 2 M_{\text {Jup }}<M<80 M_{\text {Jup }}$ and $0.01 \leq Q \leq 0.05)$, the fraction range is $[0 \%, 2.6 \%]$ for case 3 $\left(1 M_{\text {Jup }}<M<80 M_{\text {Jup }}\right.$ and $\left.Q \leq 0.01\right)$.

\subsubsection{Strengths and limitations of our approach}

Our objective is to derive the planet frequency around low-mass stars and compare this value with the frequency of planets orbiting high-mass stars (see Sect. 4). In the present analysis, we focus on the mass ratio $Q$ between the planets and their host stars instead of their masses, since the resulting $Q$ can significantly differ if considering low-mass or high-mass stars. For instance, while $Q$ is around $20 \%$ for the 2 M1207 system, its value is less than $1 \%$ for the $\beta$ Pictoris system (Lagrange et al. 2010), more than an order of magnitude smaller, while the companion masses are comparable. This issue is particularly important since planetary formation mechanisms that take place in a protostellar disc (such as CA and GI) directly depend on the disc mass and hence on the stellar mass (Andrews et al. 2013). Formation scenarios are probably more sensitive to $Q$ than to the mass of the companion. Indeed, $\mathrm{CA}$ is not expected to form planets with a mass ratio greater than a few percents (e.g., Mordasini et al. 2012). In contrast, GI has difficulties to form low $Q$ companions and tends to form only companions with higher $Q$, such as brown dwarfs or even low-mass stars (e.g., Dodson-Robinson et al. 2009; Vorobyov 2013). 
However, the mass ratio needs to be used with caution, because it sometimes corresponds to companion mass ranges where survey sensitivities are low (e.g. for $Q<0.01$, see "case 3" below, around low-mass stars). Another shortfall of using the mass ratio to extract observational constraints on companion formation mechanisms is that even though the range where each formation mechanism takes place is qualitatively clear (CA at low $Q$, GI at moderate $Q$ and stellar formation mechanisms for high $Q$ ), they would likely overlap (e.g., Reggiani et al. 2016). Nevertheless, the semi-arbitrary limits in the $Q$ interval we retain for "case 2 " $(0.01<Q<0.05)$ accommodate both our goals of probing the range of "intermediate $Q^{\prime \prime}$, and of probing the range of mass where our observational constraints are relevant. Moreover, in case 2, where $Q=0.05$ and the host star mass is $2 M_{\odot}$ (the typical mass of an A-type star), we should include companions of up to $100 \mathrm{M}_{\mathrm{Jup}}$ that are cut by our additional limit in absolute mass of $80 \mathrm{M}_{\mathrm{Jup}}$. Thus, the fraction $f$ of the most massive stars hosting SCs is underestimated for case 2. That limitation cannot be avoided when comparing planet imaging surveys of low-mass stars and more massive hosts whether or not using mass ratio. Moreover, depending on the mass ratio, the statistical significance of each observation (that is the probability to detect a companion) is taken into account, depending on the considered mass ratio. For instance, a young late-M dwarf with a mass of $0.1 M_{\odot}$ would have a high statistical significance in case 1 because contrast around faint stars is usually favorable. On the contrary, this late-M dwarf would have a very low significance for case $3(Q<0.01)$ because only planets lower than $1 M_{\text {Jup }}$ would be taken into account, and our observations are only marginally sensitive to these masses. Despite these limitations, using the $Q$ approach is an improvement over the common approach that consists of using only masses.

\section{Impact of the stellar mass on the planet frequencies}

In this section, we test the dependence of planet occurrence rate with respect to stellar mass (to identify possible population differences). To do so, we compare the planet frequencies found for the low-mass stars in MASSIVE to the ones corresponding to higher mass stars in Rameau et al. (2013b), which we derive using the same statistical formalism.

The sample that we use for comparison is made of $37 \mathrm{~A}$ to $\mathrm{F}$ type stars that are young $(<100 \mathrm{Myr})$, nearby $(<65 \mathrm{pc})$, and bright $(K<7 \mathrm{mag})^{2}$.

Both surveys (MASSIVE and A-F type stars survey) share roughly the same sample selection criteria (distance, age). The data were obtained, reduced and analyzed with the same tools so that relative biases are limited. The sensitivity of each survey is different which is properly handled by our statistical formalism see Fig. 4). In the following, we refer the MASSIVE targets as low-mass stars (hereafter LMS), and the A-F type stars as highmass stars (hereafter HMS).

\footnotetext{
2 The star around which a new planet has been imaged (51 Eri b Macintosh et al. 2015) belongs the A-F stars survey. However, the analysis led by Rameau et al. (2013b) takes into account the nondetectability of this planet (with the 2013 data) in the frequency derivation, so that this value is not affected by this new discovery.
}

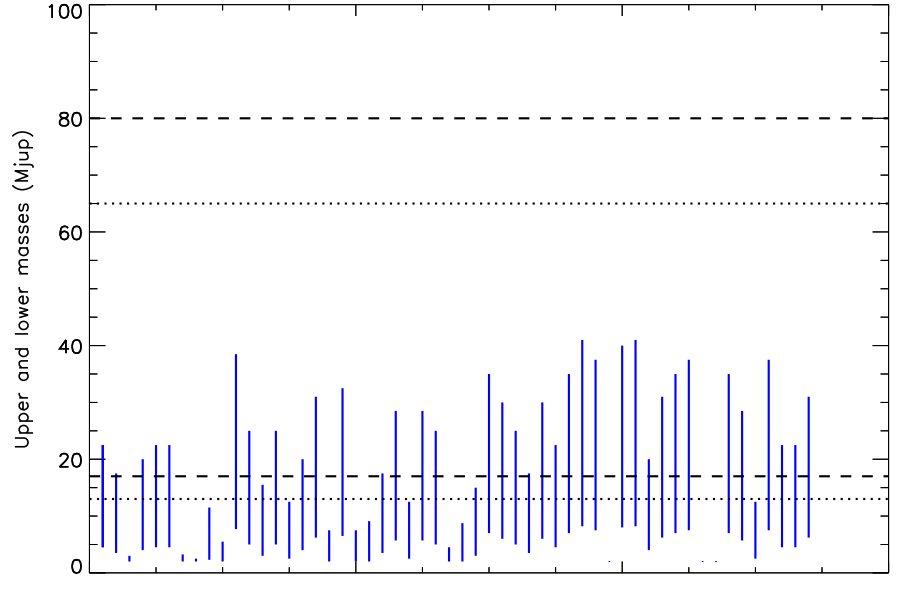

Mass ranges for MASSIVE targets, cases 2 and 3

Fig. 3. Representation of the mass ranges considered for each of our MASSIVE targets (each vertical line represents one target). The upper dashed (respectively dotted) lines represent the upper mass limit for typical A $\left(1.7 M_{\odot}\right.$; respectively F, $\left.1.3 M_{\odot}\right)$ type stars, and the lowest lines are the lower mass limits for these typical A-F type stars. We limit our study to the planet and brown dwarf mass domain, we therefore fix the upper mass limit to $80 M_{\odot}$.

\subsection{LMS and HMS companion frequency comparisons}

As a first step, we use the Kolmogorov-Smirnov (KS) test to establish if the probability distributions of the SC frequency derived from each survey are similar or not. If the KS statistic is high, it hints that the two observed companion frequency distributions originate from distinct populations. Such a difference would be indicative of different companion populations as a function of the stellar mass. We use here the KS test to compare probability distributions that artificially count thousands of stars that KS test considers as "observations". This means that the $\mathrm{KS}$ approach is only qualitatively relevant and cannot be used to provide quantitative probabilities. Deriving such quantitative probabilities is the purpose of the next section.

Case 1. Actual detections corresponding to this case are made around 2MASS0103(AB) and 2M1207 for the LMS survey, and $\beta$ Pictoris, HR 8799 and HIP 95261 for the HMS survey (cf. Fig. D.4). We find (see Fig. D.3) that $4.4_{-1.3}^{+3.2 \%}$ (at 68\% CL) of low-mass stars host at least one SC, while this fraction is $10.8_{-2.9}^{+5.7} \%$ for $\mathrm{HMS}^{3}$. This difference might be significant, as it is associated to a high $\mathrm{KS}$ statistic (0.71).

Case 2. We consider the detection of two systems; 2MASS0103(AB) for the LMS survey and HIP95261 for the HMS survey. We find (see Fig. D.3) that the $68 \%$ confidence level (CL) companion frequency ranges are [1.7\%, 5.6\%] for LMS and $[2.4 \%, 7.9 \%$ ] for HMS. We find a KS statistic of 0.23. In Fig. 3, we summarize the mass ranges considered in case 2, and the overlap between the LMS the HMS surveys: for some stars, there is very little overlap or no overlap at all, depending on their mass. This is the case for the late M dwarfs

3 The results we obtain for the HMS survey give a lower planetary frequency than those obtained by Rameau et al. (2013b). Using the same data, they found a probability of $16.1_{-5.3}^{+8.7} \%$ at $68 \%$ confidence level and considering PMCs $\left([1,13] M_{\text {Jup }}\right)$ at $[1,1000] \mathrm{AU}$, and they considered two detections, while we find a lower frequency $\left(10.8_{-2.9}^{+5.7} \%\right.$ for case 1$)$. However, the [mass, semi-major axis] domain we consider focuses only on the range of parameters where the MASSIVE sensitivity is high. Moreover the error bars on our derived frequencies are smaller since we used a natural conjugate prior in our statistical formalism. 

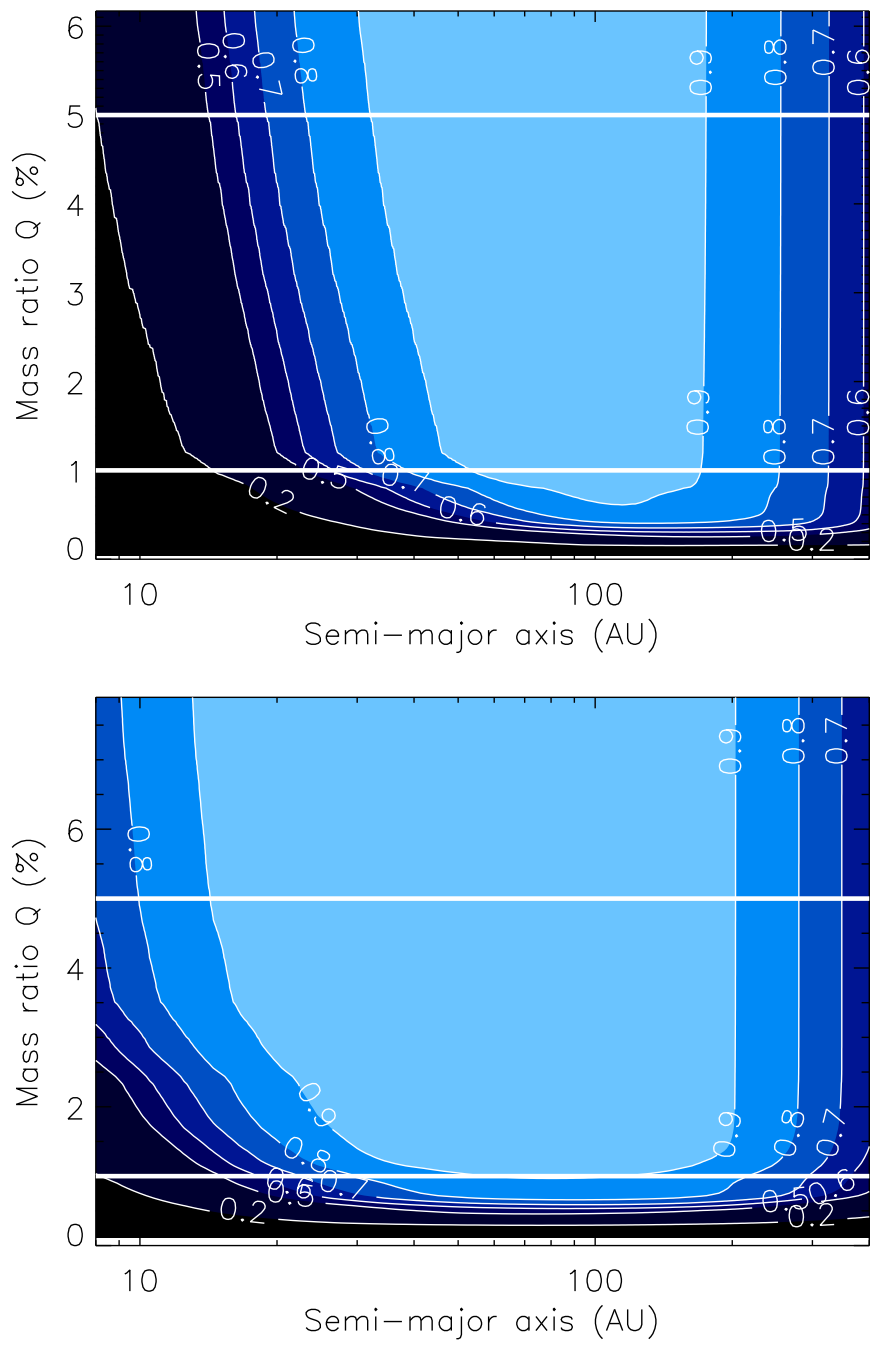

Fig. 4. Detection probability contours for the A-F survey (top) and MASSIVE (bottom) for several masses, as a function of semi-major axis. The two white lines correspond to the mass ratio limits for cases 2 and 3 (respectively $0.01<Q<0.05$ and $0.01>Q$ ).

in MASSIVE, but our statistical formalism properly handles this, hence they are included even if their actual statistical contribution is marginal or null.

Case 3. No companion in the LMS survey fulfills the conditions of case 3, and for the HMS survey we include two systems ( $\beta$ Pictoris and HR 8799). We find that both distributions are well separated (see Fig. D.3) with a KS statistic of 0.82 . We find that $9.1_{-2.7}^{+6.5} \%$ of the HMS host at least one companion (with $68 \% \mathrm{CL}$ ), while less than $2.6 \%$ (68\% CL) of the LMS host one companion.

\subsection{Relation between the SC frequency and the stellar mass}

Results presented in Sect. 4.1 seem to qualitatively hint at the existence of two distinct populations of SCs around LMS and HMS. In this section, we quantitatively investigate the following question: are there two distinct populations of SCs, one for lowmass stars, and one for more massive stars?

To mitigate possible observational biases that would artificially enhance the inferred difference, we developed a Monte Carlo code that uses contrapositive logic to provide a conservative absolute probability of whether there are two distinct populations of SCs, depending on the mass of the host star. The idea is to test if the KS statistics that we derive using our observations when comparing the MASSIVE and the A-F type star surveys are caused by the low number of detected planets, or truly distinct populations. Our rationale is the following: we assume that there is one unique underlying population of SCs by merging the two samples of stars to derive a single frequency distribution. Then, with the MC code, we test how likely it is that the random fluctuations in the observation of this single population can yield frequency distributions as different as our observations. The details of our code are described in Appendix B.

We report our results in Table D.7. We find a probability of $74.2 \%$ that there are two populations of SCs in case $1,20.6 \%$ considering case 2 , and $74.5 \%$ for case 3 .

\subsection{Discussion}

We compared the frequencies derived from the two surveys in the 3 different cases of the allowed star-planet mass ratio $Q$ described above.

Case 1 (any mass ratio is allowed). We derived SC frequencies of $4.4_{-1.3}^{+3.2} \%$ for the LMS star sample and $10.8_{-2.9}^{+5.7} \%$ for the HMS sample described in Rameau et al. (2013b). We found that in a single population there is only $\sim 26 \%$ chance to measure frequencies as distinct as we have. This translates to a $\sim 74 \%$ probability that these observations are not randomly drawn from a single population but represent two distinct planet population frequencies.

Case $2(0.01<Q<0.05)$. We tentatively associated the intermediate mass ratios of Case 2 to planets more likely formed by GI. When comparing the low-mass and high-mass star SC frequencies, we found moderately strong statistical evidence that there are not distinct populations around low-mass and high-mass stars (see Fig. D.3), since we show that there is a $\sim 79 \%$ probability that a unique SC population could reproduce the observed distribution of SCs. However, the corresponding companion masses for low-mass and high-mass stars seem to be quite different; planetary mass companions for LMSs and mostly brown dwarfs for HMSs. Our results for case 2 hints the existence at large separations of an intermediate mass-ratio population of substellar companions that is almost absent in results of surveys probing short separations and whose frequency would not be correlated with stellar mass. However, our contrapositive logical approach starts by assuming the existence of such a single population. Therefore, the probability derived for the existence of two populations are conservative by construct (as for cases 1 and 3), while on the opposite the $\sim 79 \%$ of a unique population for case 2 is not. Our results only suggest that the existence of two distinct populations is not necessary to explain the data considered for case 2 .

Case 3 ( $Q \leq 0.01$ ). We found a $\sim 75 \%$ probability that two distinct populations of companions exist around LMSs and HMSs.

Given the sensitivity of our survey, we cannot exclude the possibility that less massive giant planets would be as frequent around LMSs as around HMSs. It is known that low-mass planets such as Neptune-like planet masses that form the bulk of gas-giant population around LMSs at short separations (see e.g., Bonfils et al. 2013), and a substantial population of super-Earths to Jupiter-mass planets are detected at moderate separations ( 0.5-10 AU) around LMSs using micro-lensing method (Cassan et al. 2012). Theoretical models of CA also indicate that the formation of giant planets at small separations 
by CA is difficult around LMSs (Mordasini et al. 2012). If the same is true at large separations, then the frequency derived in this work are significantly lower than the actual frequency when the Neptune-like planets are also considered.

\section{Comparison with previous studies}

We used a conjugate Bayesian prior which qualitatively assumes that since we found few giant exoplanets in the range we selected, low frequencies of exoplanets are more probable than high frequencies. This approach is different from that of Bowler et al. (2015) because $100 \%$ of our targets have been followed-up, providing perfect completeness in our subsequent statistical analysis which is not the case in Bowler et al. (2015). In contrast with this study, we found some statistical evidence for a correlation between the SC frequency and the stellar mass at large separations.

It is difficult to compare our results with those from other surveys because most of the time the parameter ranges considered are not exactly the same. We therefore applied our formalism by considering exactly the same domains of mass and semi-major axis as those probed by each of RV and DI (Montet et al. 2014), micro lensing and RV (Clanton \& Gaudi 2014), and RV surveys (Bonfils et al. 2013). Table D.5 shows our companion frequencies for these ranges and those derived within these surveys. For our calculations, we considered a $0.5 M_{\text {Sun }}$ star and a inclination of $60^{\circ}$. Our results are consistent with results described by Clanton \& Gaudi (2014) and Bonfils et al. (2013). They reveal a tension at $1.5 \sigma$ with the results of Montet et al. (2014), that is of marginal significance.

\section{Conclusion}

We conducted an adaptive optics survey for exoplanets orbiting nearby young low mass stars using $L^{\prime}$-band observations with $\mathrm{NaCo}$ at VLT. Two planetary mass companions were detected and previously reported (Delorme et al. 2013; Chauvin et al. 2004), but no additional companions were detected. From our significant data set (54 targets), we derived the planetary mass companion (and substellar companion) frequency, defined here by the probability that a star hosts at least one planetary (substellar) mass companion. We used Bayesian statistics with an optimized conjugate prior, and we selected the sub-range in planet mass and semi-major axes where our data provides the best information, so that our statistics does not consider parameter ranges where our survey is not sensitive to companions. Our range of interest corresponds to planets more massive than $2 M_{\text {Jup }}$ and a semi-major axis between 8 and $400 \mathrm{AU}$. Using this range, we determined with a $68 \%$ confidence level that substellar companion frequency is $4.4_{-1.3}^{+3.2} \%$, and that planetary mass companion frequency is $2.3_{-0.7}^{+2.9} \%$. Considering all companions regardless of planet-to-star mass ratio, a Monte Carlo comparison between the substellar companion frequencies of the low-mass stars in the MASSIVE survey and a higher-mass A-F type star survey shows that there is a $\sim 74 \%$ probability that there are two distinct substellar companion populations, one orbiting around the low-mass stars and one orbiting around the higher-mass A-F type stars. We also found a $\sim 75 \%$ probability considering only low-mass ratios $(Q<1 \%)$. These results suggest that the frequency of imaged wide-orbit substellar companions is correlated with the stellar mass, in agreement with theoretical expectations (see e.g., Laughlin et al. 2004). For intermediate mass ratios $(1 \%<Q<5 \%)$, the same comparison indicates a statistically significant similarity between the substellar companion populations around the low-mass star and high-mass star samples. We acknowledge that our results showing that substellar companion frequency could be correlated to stellar mass are still moderately significant. We therefore need more observations to confirm this important result.

Acknowledgements. We thank the staff of ESO-VLT for their support at the telescope. We acknowledge support from the French National Research Agency (ANR) through the GuEPARD project grant ANR10-BLANC0504-01. We acknowledge financial support from "Programme National de Physique Stellaire" (PNPS) of CNRS/INSU, France. We thank Didier Fraix-Burnet for our discussions on statistics. The research of J.E.S. was supported by an appointment to the NASA Postdoctoral Program at NASA Ames Research Center, administered by Oak Ridge Associated Universities through a contract with NASA.

\section{References}

Adibekyan, V. Z., Figueira, P., Santos, N. C., et al. 2013, A\&A, 560, A51

Alibert, Y., Mordasini, C., \& Benz, W. 2004, A\&A, 417, L25

Allard, F., Homeier, D., \& Freytag, B. 2012, Roy. Soc. Lond. Philosoph. Trans. Ser. A, 370, 2765

Allers, K. N., \& Liu, M. C. 2013, ApJ, 772, 79

Allers, K. N., Jaffe, D. T., van der Bliek, N. S., Allard, F., \& Baraffe, I. 2006, ASP Conf. Ser. 357, eds. L. Armus, \& W. T. Reach, 77

Andrews, S. M., Rosenfeld, K. A., Kraus, A. L., \& Wilner, D. J. 2013, ApJ, 771, 129

Artigau, É., Gagné, J., Faherty, J., et al. 2015, ApJ, 806, 254

Baraffe, I., Chabrier, G., Barman, T. S., Allard, F., \& Hauschildt, P. H. 2003, A\&A, 402, 701

Barenfeld, S. A., Bubar, E. J., Mamajek, E. E., \& Young, P. A. 2013, ApJ, 766, 6 Béjar, V. J. S., Osorio, M. R. Z., Pérez-Garrido, A., et al. 2009, in 15th Cambridge Workshop on Cool Stars, Stellar Systems, and the Sun, ed. E. Stempels, AIP Conf. Ser., 1094, 469

Binks, A. S., \& Jeffries, R. D. 2014, MNRAS, 438, L11

Bonavita, M., Chauvin, G., Desidera, S., et al. 2012, A\&A, 537, A67

Bonavita, M., Desidera, S., Thalmann, C., et al. 2016, A\&A, 593, A38

Bonfils, X., Delfosse, X., Udry, S., et al. 2013, A\&A, 549, A109

Bonnefoy, M., Marleau, G.-D., Galicher, R., et al. 2014, A\&A, 567, L9

Boss, A. P. 2011, ApJ, 731, 74

Bowler, B. P., Liu, M. C., Shkolnik, E. L., \& Tamura, M. 2015, ApJS, 216, 7

Burgasser, A. J., Simcoe, R. A., Bochanski, J. J., et al. 2010, ApJ, 725, 1405

Cameron, A. G. W. 1978, Moon and Planets, 18, 5

Carson, J. C., Kern, B. D., Trauger, J. T., \& Breckinridge, J. B. 2006, in SPIE Conf. Ser., 6265

Cassan, A., Kubas, D., Beaulieu, J.-P., et al. 2012, Nature, 481, 167

Chauvin, G., Lagrange, A.-M., Dumas, C., et al. 2004, A\&A, 425, L29

Chauvin, G., Lagrange, A.-M., Dumas, C., et al. 2005, A\&A, 438, L25

Chauvin, G., Lagrange, A.-M., Bonavita, M., et al. 2010, A\&A, 509, A52

Clanton, C., \& Gaudi, B. S. 2014, ApJ, 791, 91

Currie, T., Daemgen, S., Debes, J., et al. 2014, ApJ, 780, L30

Daemgen, S., Siegler, N., Reid, I. N., \& Close, L. M. 2007, ApJ, 654, 558

Delorme, P., Lagrange, A. M., Chauvin, G., et al. 2012, A\&A, 539, A72

Delorme, P., Gagné, J., Girard, J. H., et al. 2013, A\&A, 553, L5

Dodson-Robinson, S. E., Veras, D., Ford, E. B., \& Beichman, C. A. 2009, ApJ, 707, 79

Ducourant, C., Teixeira, R., Galli, P. A. B., et al. 2014, A\&A, 563, A121

Dupuy, T. J., Liu, M. C., \& Ireland, M. J. 2014, ApJ, 790, 133

Elliott, P., Huélamo, N., Bouy, H., et al. 2015, A\&A, 580, A88

Fuhrmeister, B., \& Schmitt, J. H. M. M. 2003, A\&A, 403, 247

Gagné, J., Faherty, J. K., Cruz, K., et al. 2014a, ApJ, 785, L14

Gagné, J., Lafrenière, D., Doyon, R., Malo, L., \& Artigau, É. 2014b, ApJ, 783, 121

Gagné, J., Faherty, J. K., Cruz, K. L., et al. 2015a, ApJS, 219, 33

Gagné, J., Lafrenière, D., Doyon, R., Malo, L., \& Artigau, É. 2015b, ApJ, 798, 73

Gauza, B., Béjar, V. J. S., Pérez-Garrido, A., et al. 2015, ApJ, 804, 96

Ireland, M. J., Kraus, A., Martinache, F., Law, N., \& Hillenbrand, L. A. 2011, ApJ, 726, 113

Itoh, Y., Hayashi, M., Tamura, M., et al. 2005, ApJ, 620, 984

Kasper, M., Apai, D., Janson, M., \& Brandner, W. 2007, A\&A, 472, 321

Kastner, J. H., Huenemoerder, D. P., Schulz, N., et al. 1997, BAAS, 29, 1361

Kraus, A. L., Ireland, M. J., Cieza, L. A., et al. 2014a, ApJ, 781, 20

Kraus, A. L., Shkolnik, E. L., Allers, K. N., \& Liu, M. C. 2014b, AJ, 147, 146

Kuzuhara, M., Tamura, M., Kudo, T., et al. 2013, ApJ, 774, 11 
Lafrenière, D., Marois, C., Doyon, R., Nadeau, D., \& Artigau, É. 2007, ApJ, 660, 770

Lagrange, A.-M., Bonnefoy, M., Chauvin, G., et al. 2010, Science, 329, 57 Lambrechts, M., \& Johansen, A. 2012, A\&A, 544, A32

Laughlin, G., Bodenheimer, P., \& Adams, F. C. 2004, ApJ, 612, L73

Lenzen, R., Hartung, M., Brandner, W., et al. 2003, in Instrument Design and Performance for Optical/Infrared Ground-based Telescopes, eds. M. Iye, \&

A. F. M. Moorwood, SPIE Conf. Ser., 4841, 944

Lowrance, P. J., Schneider, G., Kirkpatrick, J. D., et al. 2000, ApJ, 541, 390

Luhman, K. L., Stauffer, J. R., \& Mamajek, E. E. 2005, ApJ, 628, L69

Luhman, K. L., Wilson, J. C., Brandner, W., et al. 2006, ApJ, 649, 894

Luhman, K. L., Mamajek, E. E., Allen, P. R., Muench, A. A., \& Finkbeiner, D. P. 2009, ApJ, 691, 1265

Macintosh, B., Graham, J. R., Barman, T., et al. 2015, Science, 350, 64

Makarov, V. V., \& Urban, S. 2000, MNRAS, 317, 289

Malo, L., Doyon, R., Lafrenière, D., et al. 2013, ApJ, 762, 88

Malo, L., Artigau, É., Doyon, R., et al. 2014a, ApJ, 788, 81

Malo, L., Doyon, R., Feiden, G. A., et al. 2014b, ApJ, 792, 37

Mamajek, E. E., \& Bell, C. P. M. 2014, MNRAS, 445, 2169

Marois, C., Lafrenière, D., Doyon, R., Macintosh, B., \& Nadeau, D. 2006, ApJ, 641, 556

Marois, C., Macintosh, B., Barman, T., et al. 2008, Science, 322, 1348

Marois, C., Macintosh, B., \& Véran, J.-P. 2010, in SPIE Conf. Ser., 7736

Mayor, M., \& Queloz, D. 1995, Nature, 378, 355

Montet, B. T., Crepp, J. R., Johnson, J. A., Howard, A. W., \& Marcy, G. W. 2014, ApJ, 781, 28

Mordasini, C., Alibert, Y., Benz, W., Klahr, H., \& Henning, T. 2012, A\&A, 541, A97

Naud, M.-E., Artigau, É., Malo, L., et al. 2014, ApJ, 787, 5

Pollack, J. B., Hubickyj, O., Bodenheimer, P., et al. 1996, Icarus, 124, 62

Pueyo, L., Soummer, R., Hoffmann, J., et al. 2015, ApJ, 803, 31

Rameau, J., Chauvin, G., Lagrange, A.-M., et al. 2013a, ApJ, 772, L15
Rameau, J., Chauvin, G., Lagrange, A.-M., et al. 2013b, A\&A, 553, A60 Reggiani, M., Meyer, M. R., Chauvin, G., et al. 2016, A\&A, 586, A147 Riaz, B., Gizis, J. E., \& Harvin, J. 2006, AJ, 132, 866

Rice, E. L., Barman, T., Mclean, I. S., Prato, L., \& Kirkpatrick, J. D. 2010, ApJS, 186, 63

Riedel, A. R., Finch, C. T., Henry, T. J., et al. 2014, AJ, 147, 85

Riedel, A. R., et al. 2016, AJ, submitted

Rodriguez, D. R., Zuckerman, B., Kastner, J. H., et al. 2013, ApJ, 774, 101

Rousset, G., Lacombe, F., Puget, P., et al. 2003, in Adaptive Optical System Technologies II, eds. P. L. Wizinowich, \& D. Bonaccini, SPIE Conf. Ser., 4839, 140

Schlieder, J. E. 2011, Ph.D. Thesis, State University of New York at Stony Brook, 73

Schlieder, J. E., Lépine, S., \& Simon, M. 2012, AJ, 144, 109

Schneider, A., Song, I., Melis, C., Zuckerman, B., \& Bessell, M. 2012, ApJ, 757, 163

Shkolnik, E. L., Liu, M. C., Reid, I. N., Dupuy, T., \& Weinberger, A. J. 2011, ApJ, 727, 6

Shkolnik, E. L., Anglada-Escudé, G., Liu, M. C., et al. 2012, ApJ, 758, 56

Soummer, R., Pueyo, L., \& Larkin, J. 2012, ApJ, 755, L28

Todorov, K., Luhman, K. L., \& McLeod, K. K. 2010, ApJ, 714, L84

Torres, C. A. O., Quast, G. R., Melo, C. H. F., \& Sterzik, M. F. 2008, Young Nearby Loose Associations, ed. Reipurth, B., 757

Udalski, A., Zebrun, K., Szymanski, M., et al. 2002, Acta Astron., 52, 115

Vigan, A., Patience, J., Marois, C., et al. 2012, A\&A, 544, A9

Vorobyov, E. I. 2013, A\&A, 552, A129

Wahhaj, Z., Liu, M. C., Biller, B. A., et al. 2011, ApJ, 729, 139

Webb, R. A., \& Zuckerman, B. 1999, BAAS, 31, 1416

Weinberger, A. J., Anglada-Escudé, G., \& Boss, A. P. 2013, ApJ, 762, 118

Wolszczan, A., \& Frail, D. A. 1992, Nature, 355, 145

Zuckerman, B., \& Webb, R. A. 2000, ApJ, 535, 959

Zuckerman, B., Song, I., \& Bessell, M. S. 2004, ApJ, 613, L65 


\section{Appendix A: Statistical analysis}

Our statistical formalism is based on works by Carson et al. (2006), Lafrenière et al. (2007), Vigan et al. (2012), and Rameau et al. (2013b).

Let us consider $N$, the total number of stars in a survey. From our detection limit analysis described in the text, the mean detection probability to find a companion of a given mass at a given semi-major axis, around a given target $j$ of a survey is $p_{j} . p_{j}$ is derived from the $2 \mathrm{D}$ detection limit maps. We denote $f$ the fraction of stars around which there is at least one planet, with a mass included in the interval $\left[m_{\min }, m_{\max }\right]$, and for separations inside the interval $\left[a_{\min }, a_{\max }\right] . f p_{j}$ is the probability to detect a PMC around the star $j$ given its mean detection probability $p_{j}$ and the fraction of stars $f$, and $1-f p_{j}$ is the probability not to find it. The detections and non-detections that are reported for a survey are denoted $\left\{d_{j}\right\}: d_{j}=1$ for stars around which we found a planet, and 0 otherwise. The likelihood function of the data from which we can find $f$ is $L\left(\left\{d_{j}\right\} \mid f\right)$, which is the product of each Bernoulli event: this represents the probability model that gives $\left\{d_{j}\right\}$ given the planet fraction $f$.

$L\left(\left\{d_{j}\right\} \mid f\right)=\prod_{j=1}^{N}\left(1-f p_{j}\right)^{1-d_{j}} \times\left(f p_{j}\right)^{d_{j}}$

Bayes' theorem provides the probability density of $f$, the fraction of stars hosting at least one PMC given the observed data $\left\{d_{j}\right\}$ : this probability density is the posterior distribution that represents the distribution of $f$ given the observed data $\left\{d_{j}\right\}$.

$P\left(f \mid\left\{d_{j}\right\}\right)=\frac{L\left(\left\{d_{j}\right\} \mid f\right) P(f)}{\int_{0}^{1} L\left(\left\{d_{j}\right\} \mid f\right) P(f) \mathrm{d} f}$.

Where $P\left(\left\{d_{j}\right\}\right)=\int_{0}^{1} L\left(\left\{d_{j}\right\} \mid f\right) P(f) \mathrm{d} f$ is called the marginalized likelihood.

Once $f$ is calculated, we determine the interval $\left[f_{\min }, f_{\max }\right]$ of $f$ for a confidence level $C L$ as:

$C L=\int_{f_{\min }}^{f_{\max }} P\left(f \mid\left\{d_{j}\right\}\right) \mathrm{d} f$

An easier way to find $f_{\min }$ and $f_{\max }$ is to split the former equation into two others:

$$
\begin{aligned}
& \frac{1-C L}{2}=\int_{f_{\max }}^{1} P\left(f \mid\left\{d_{j}\right\}\right) \mathrm{d} f \\
& \frac{1-C L}{2}=\int_{0}^{f_{\min }} P\left(f \mid\left\{d_{j}\right\}\right) \mathrm{d} f
\end{aligned}
$$

In case of a null detection, the likelihood function is approximated using Poisson statistics:

$L\left(\left\{d_{j}\right\} \mid f\right)=\prod_{j=1}^{N} \mathrm{e}^{-f p_{j}}$.

Moreover, for such a null detection, $f_{\min }=0$ and $f_{\max }$ is given by:

$f_{\max }=\frac{-\ln (1-C L)}{N\left\langle p_{j}\right\rangle}$.

In the Eq. (4), $P(f)$ is called the prior distribution. This prior is a distribution reporting any preexisting belief concerning the distribution of $f$. A first approach is to consider the prior distribution as a uniform distribution, $P(f)=1$. Another possibility is to define $P(f)$ as the natural conjugate prior of $P\left(f \mid\left\{d_{j}\right\}\right)$ : in that case, the prior and posterior distributions are from the same distribution family. Here, the posterior distribution is a Bernoulli distribution represented by a Beta function B:

$P(f)=\frac{\left(f p_{j}\right)^{\alpha-1}\left(1-f p_{j}\right)^{\beta-1}}{\mathrm{~B}(\alpha, \beta)}$

$\alpha$ and $\beta$ are hyperparameters that can be fixed. We can then choose these hyperparameters such that $P(f)$ is mathematically identical to $L\left(\left\{d_{j}\right\} \mid f\right)$ :

$P(f)=L\left(\left\{d_{j}\right\} \mid f\right) \propto \prod_{j=1}^{N}\left(1-f p_{j}\right)^{1-d_{j}} \times\left(f p_{j}\right)^{d_{j}}$.

Even if the mathematical forms of $P(f)$ and $L\left(\left\{d_{j}\right\} \mid f\right)$ are identical, $P(f)$ is a continuous function of $f$ given $\left\{d_{j}\right\}$, while $L\left(\left\{d_{j}\right\} \mid f\right)$ is a discrete function of $\left\{d_{j}\right\}$ given $f$.

\section{Appendix B: Details on Monte Carlo methods using contrapositive logic}

The following steps describe our Monte Carlo approach to determine the probability of a single giant planet population around the studied samples of low-mass and high-mass stars.

0/ Before running our MC code, we calculate the KolmogorovSmironov statistic for each case (see Table D.7).

1/ First, we consider for each case both surveys and all the detections we have as if LMS and HMS belonged to the same survey. Thus, we consider here only one population of SCs. We then derive the frequency of SCs for each case (cf. Fig. D.4).

2/ Second, using the merged distribution derived in $1 /$, we chose randomly the frequency $f$, for each case, that corresponds to the frequency of stars hosting at least one SC. We do it for 10000 iterations.

3/ Then, we attribute randomly to each star (for both surveys) zero or at least one substellar companion, using the frequency derived in $2 /$.

4/ If at least one substellar companion has been attributed to a star, we calculate if it has been detected using the mean probability to detect it around the considered star. Thus, after allocating each star to the survey it initially belonged to, each survey ends with $n_{1}$ and $n_{2}$ detections (respectively for the LMS and the HMS surveys).

5/ Once each original survey has been attributed $n_{1}$ and $n_{2}$ detections, we derive the frequency of SCs for the LMS and the HMS surveys, as we did previously. Then, we find two frequency ranges (for the LMS and the HMS surveys), that we will use in 6/ to know the extent to which both distributions, and thus populations, are different.

6/ We derive the Kolmogorov-Smironov statistic and its associated probability for all the iterations, for each case, using the frequency ranges found in 5/. We then count how many times the KS statistic is higher than the KS statistic derived for each case (cf. Table D.7): thus, we derive the probability $P$ that we can identify two distinct populations of SCs by chance when only one population would actually exist.

7/ We derive then the probability that there are two distinct populations of SCs when considering two distinct surveys at the beginning $(1-P)$. 


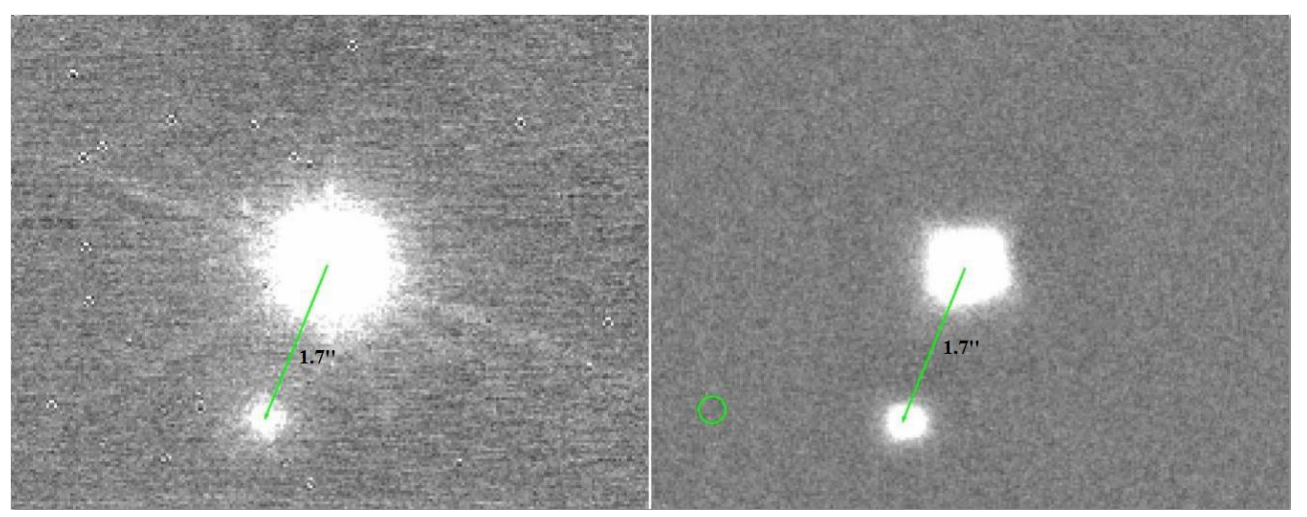

Fig. C.1. Left: J0854 A and B data from January 2006, using NaCo and the $K_{\mathrm{S}}$ band. Right: J0854 A and B in November 2012, using NaCo in the $L^{\prime}$ band. The green arrow indicates the position of the companion in 2006, and the green circle displays the expected position of J0854 (AB) C if it were a background field star. East is left and North is up.

Table C.1. Radial velocities $\left(\mathrm{km} \mathrm{s}^{-1}\right)$ obtained with HARPS.

\begin{tabular}{cccc}
\hline \hline Observation dates & Primary RV & Secondary RV & $V_{\gamma}$ \\
\hline $2013-12-05$ & 82.9 & 4.3 & 44.6 \\
$2014-02-16(1)$ & 28.4 & 62.9 & 45.2 \\
$2014-02-16(2)$ & 2.7 & 89.3 & 44.9 \\
\hline
\end{tabular}

\section{Appendix C: 2MASS J08540240-3051366}

This target was identified as a probable young star by FS2003 (Fuhrmeister \& Schmitt 2003). We used the BANYAN II Bayesian analysis tool (Gagné et al. 2015b) to assess whether it is a probable member of a young moving group. Using the proper motion that we measured from the 2MASS and AllWISE astrometry epochs $\left(\mu_{\alpha} \cos \delta=-275.3 \pm 5.5 ; \mu_{\delta}=-14.7 \pm 6.1\right)$ with its sky position and 2MASS and AllWISE photometry, we obtained a $89 \%$ membership probability to the $\beta$ Pictoris moving group (BPMG), associated with a $38 \%$ probability for it to be an unresolved binary star. The statistical distance and radial velocity (RV) associated with a membership to $\beta$ Pictoris are $10.9 \pm 1.2 \mathrm{pc}$ and $15.6 \pm 1.7 \mathrm{~km} \mathrm{~s}^{-1}$, respectively.

We observed the star with NaCo in November. 2012 (090.C0698(A)) and obtained additional $\mathrm{NaCo}$ observations from the ESO archive observed in January 2006. After data reduction and analysis, we identified a comoving companion orbiting this target (see Fig. C.1). We measured absolute magnitudes of $L^{\prime}=$ $9.50 \pm 0.05, K_{\mathrm{S}}=10.03 \pm 0.01, H=10.44 \pm 0.01$, and $J=11.05 \pm 0.03$ for the companion, which orbits at a separation of $\approx 26 \mathrm{AU}$ from its host $\operatorname{star}(\approx 1.7$ as with a position angle of $158.7 \pm 0.3^{\circ}$ from the $L^{\prime}$-band data, and $156.2 \pm 0.3^{\circ}$ from the $K_{\mathrm{S}}$-band data). The NIR colors of the companion are both consistent with a field-age or a young object, hence the photometric $\mathrm{NaCo}$ data cannot further constrain its age.

We subsequently obtained NIR spectra for the host and companion using FLAMINGOS II (GS-2013B-Q-79, PI: J. Gagné) and SINFONI (292.C-5036A, PI: J. Lannier), which allowed us to assign a spectral type of M4 to the host star. This NIR spectrum did not allow us to place constraints on the age of the host star. Contrary to the BANYAN II prediction according to which the companion would be a probable young object near the planetary-mass boundary, its NIR spectrum is similar to M8 field stars, with no apparent signs of low gravity. The spectral types mentioned above were obtained using the visual classification method described by Gagné et al. (2015b, BANYAN VII), and are both associated with an uncertainty of 0.5 subtypes.
In addition to the observations mentioned above, we obtained three high-resolution optical spectra $(R \simeq 115000$; $3800-6900 \AA$ ) of the host star with the HARPS spectrograph located at the $3.6 \mathrm{~m}$ ESO telescope at La Silla observatory (LP192.C-0224, PI: Lagrange), in order to measure its systemic RV and improve our BANYAN II membership probability assessment. We used the automated HARPS Data Reduction System to obtain a first RV measurement estimate, and identified high-amplitude variations of the order of several tens of $\mathrm{km} \mathrm{s}^{-1}$ in the RV signal. The cross-correlation functions (CCF) associated with these spectra show two peaks, indicative of a doublelined spectroscopic binary (SB2). The $\mathrm{H}_{\alpha}$ emission line also displays a double-peak shape.

We found that the two peaks of the CCF display significantly different depths, which allowed us to distinguish between the two components of the SB2 host star and to retrieve individual RV measurements. These values are summarized Table C.1. For each component, the measurement RV corresponds to the sum of the absolute RV of the binary $V_{\gamma}$ and that of the relative RV of the component with respect to $V_{\gamma}$. The relative RV is proportional to the phase of the binary system motion; we can thus obtain measurement of $V_{\gamma}$ using each spectrum. The values that we obtain are summarized in Table C.1. We take the mean value of these three systemic RV measurement to obtain $V_{\gamma}=45 \pm 1 \mathrm{~km} \mathrm{~s}^{-1}$, where the error is an empirical estimate.

These new observations strongly reject any possible membership to BPMG, both in terms of age and kinematics. The lack of low-gravity features in the NIR spectrum of the M8-type companion 2MASS J0854 (AB) C constraints the age of the system at $\gtrsim 250$ Myr (Allers \& Liu 2013), whereas the systemic RV measurements reduces the Bayesian probability of a membership to any young moving group to zero using BANYAN II (i.e., TW Hydrae, BPMG, Tucana-Horologium, Carina, Columba, Argus, and AB Doradus). We have used a new Bayesian analysis tool that includes more moving groups and associations (BANYAN-M; J. Gagné et al., in prep., see also A. Riedel et al. 2016) to further reject any possible membership to the 
Hercules-Lyrae, Carina-Near, Ursa Major, 32 Ori, Alessi 13, $\varepsilon$ Cha, $\eta$ Cha, Coma Berenices associations and moving groups, as well as to the Pleiades association.

The binary nature of the host star 2MASS J0854 (AB) reconciles the spectral type of the companion that seemed too early with respect to its contrast ratio to the host star. This system is thus in all likelihood a field-age SB2 binary M4-type host star with a wide-separation M8-type stellar companion that display a proper motion similar to BPMG members by pure chance. Such interlopers are expected in BANYAN II candidate members (e.g., see Gagné et al. 2014a, BANYAN II), which outlines the necessity of obtaining full kinematics and youth indications before declaring an object as a new bona fide member of a young moving group. We have thus excluded the 2MASS J0854 (AB) C system from our survey statistic analysis described in Sect. 3.

\section{Appendix D: Additional material}

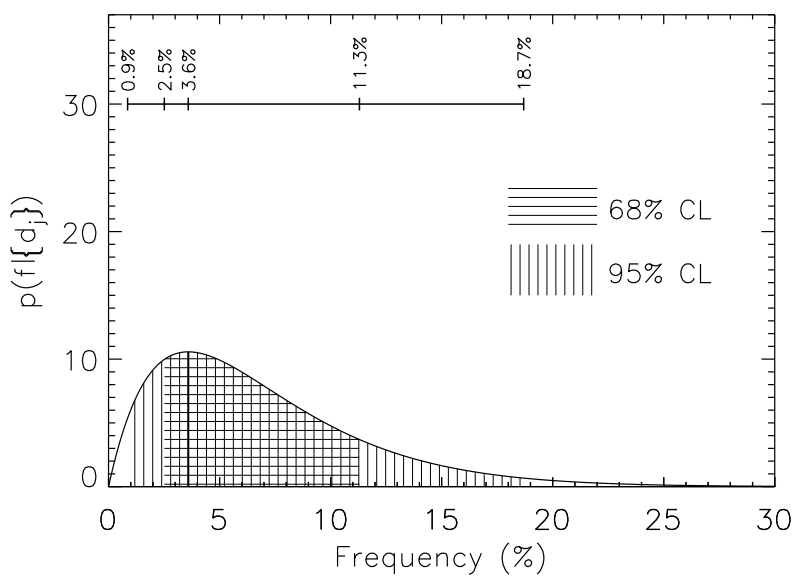

(a) Uniform prior, $[0.5,80] M_{\text {Jup }}$ and $[0.5,1000] \mathrm{AU}$.

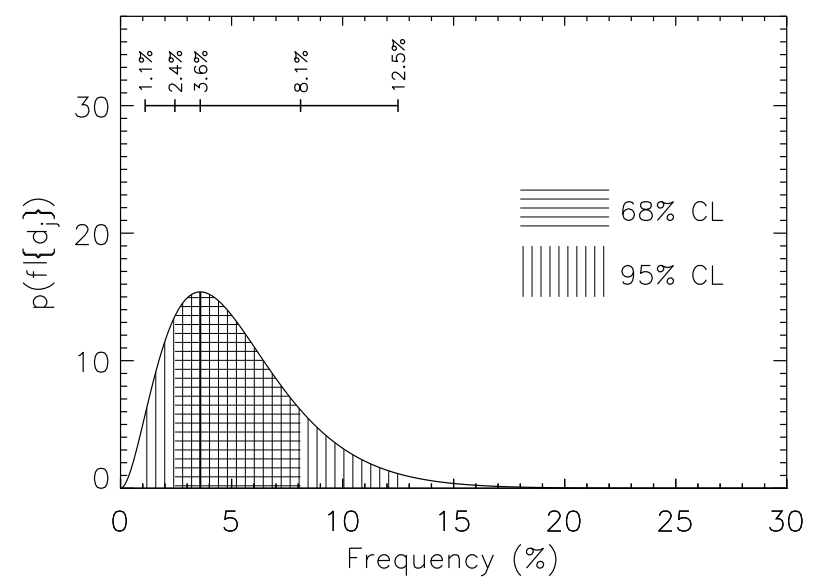

(c) Conjugate prior, $[0.5,80] M_{\text {Jup }}$ and $[0.5,1000] \mathrm{AU}$.

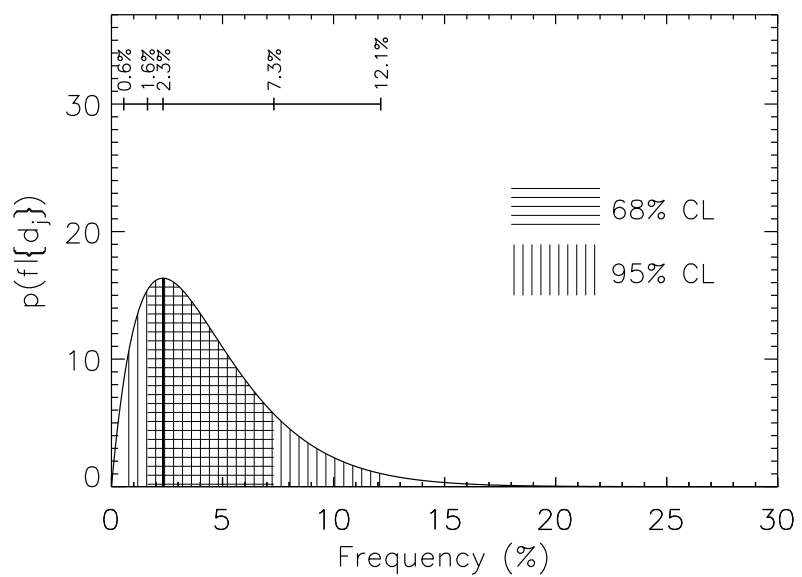

(b) Uniform prior, [2,14] $M_{\text {Jup }}$ and $[8,400] \mathrm{AU}$.

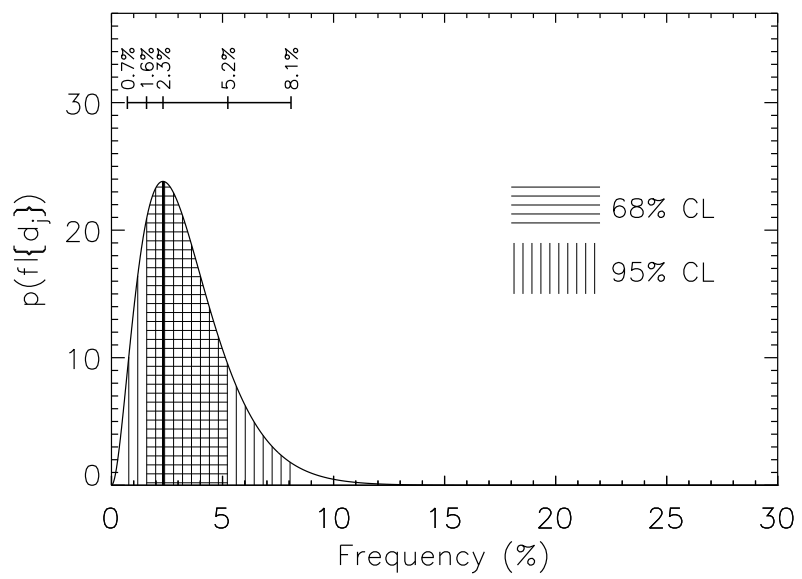

(d) Conjugate prior, [2,14] $M_{\text {Jup }}$ and [8,400] AU.

Fig. D.1. Fractions of low-mass stars in MASSIVE hosting SCs (left) and PMCs (right) for different [mass, SMA] ranges, and priors. We take into account the only detection of a PMC around 2MASS0103(AB). 


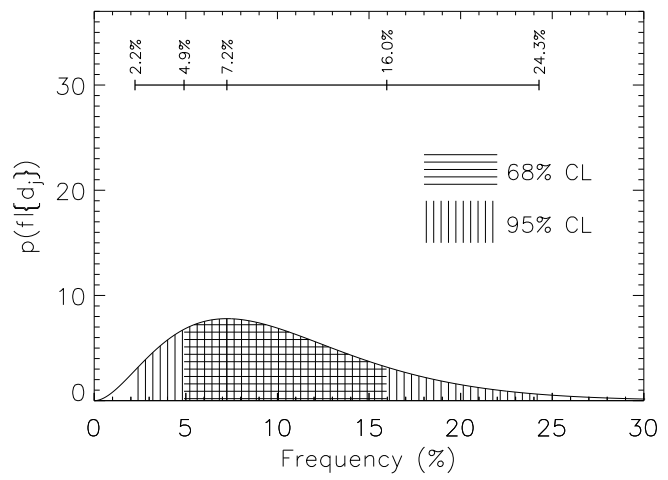

(a) Uniform prior, $[0.5,80] M_{\text {Jup }}$ and $[0.5,1000] \mathrm{AU}$.

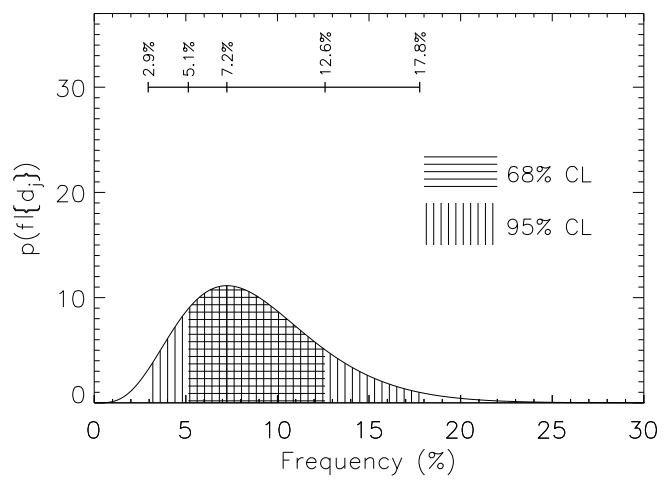

(c) Conjugate prior, $[0.5,80] M_{\text {Jup }}$ and $[0.5,1000] \mathrm{AU}$.

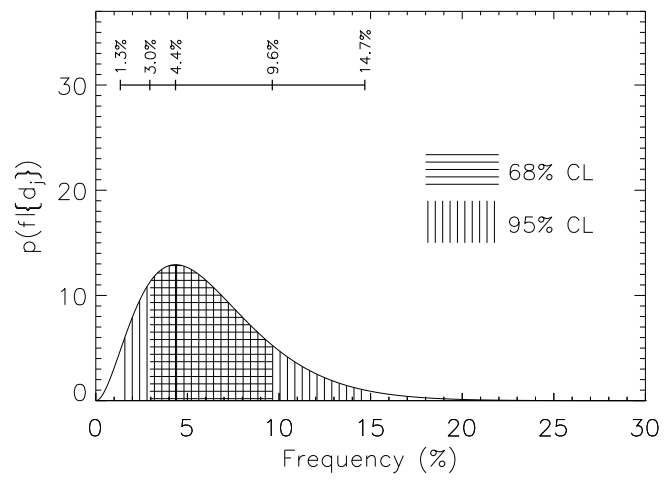

(b) Uniform prior, $[2,80] M_{\text {Jup }}$ and $[8,400] \mathrm{AU}$.

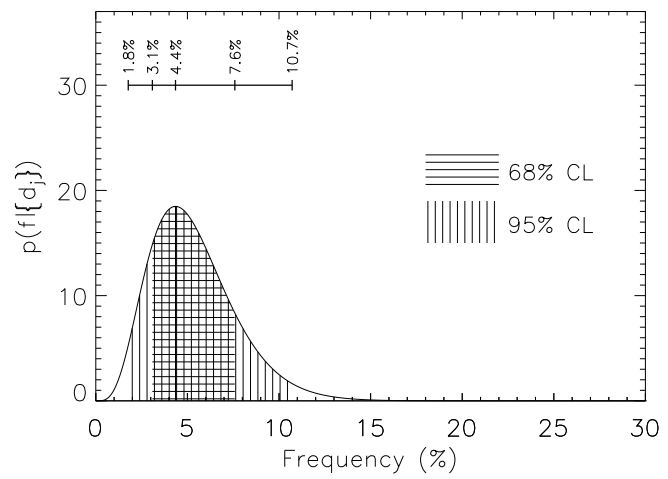

(d) Conjugate prior, $[2,80] M_{\text {Jup }}$ and $[8,400] \mathrm{AU}$

Fig. D.2. Fractions of low-mass stars in MASSIVE hosting SCs for different [mass, SMA] ranges, and priors. We take into account the detection of a PMC around 2MASS0103(AB) and 2M1207.

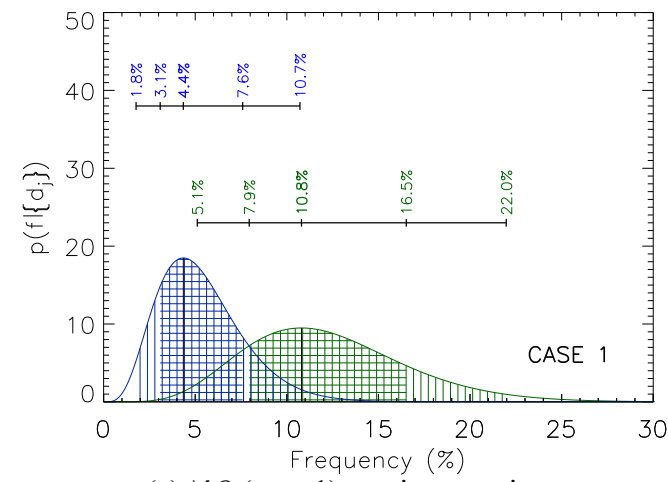

(a) $\forall Q$ (case 1), conjugate prior

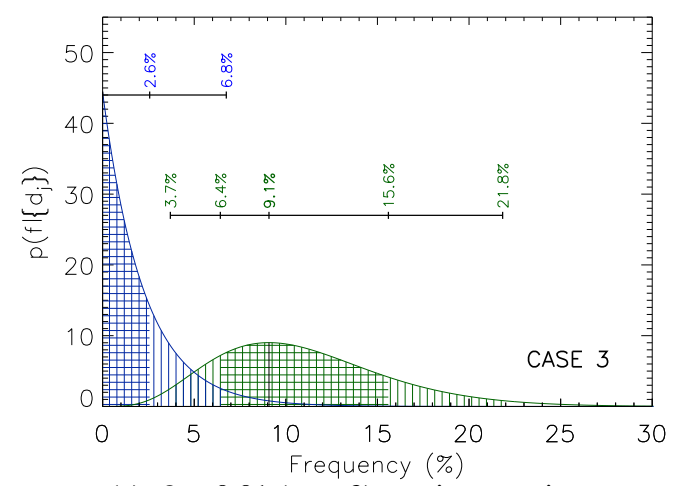

(c) $Q<0.01$ (case 3), conjugate prior

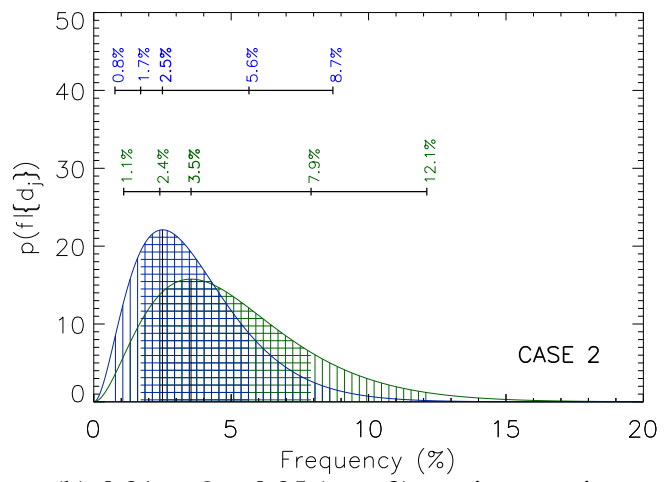

(b) $0.01<Q<0.05$ (case 2), conjugate prior

Fig. D.3. Comparisons of the frequency distributions as function of stellar mass calculated for the three cases described in the text, considering low-mass (blue) and higher mass (green) stars. 
J. Lannier et al.: MASSIVE: A Bayesian analysis of giant planet populations around low-mass stars

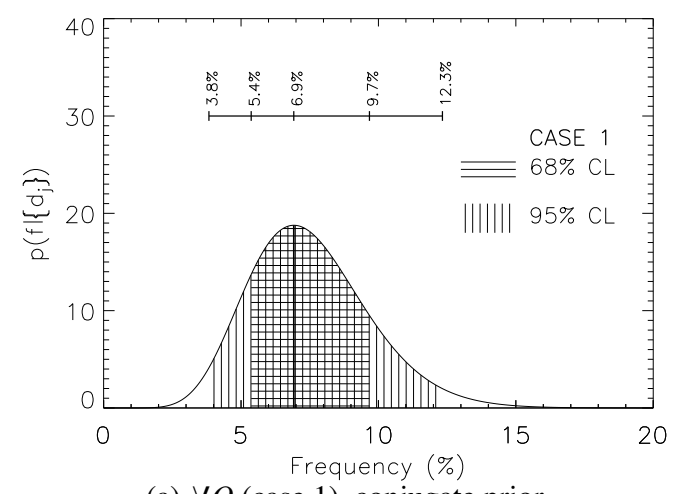

(a) $\forall Q$ (case 1), conjugate prior

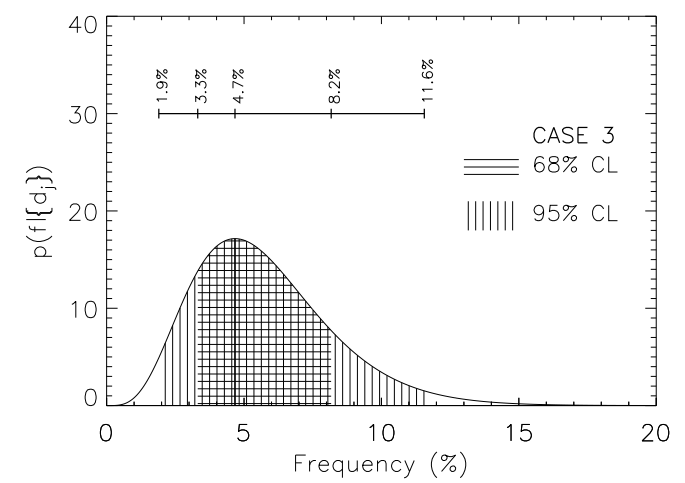

(c) $Q<0.01$ (case 3), conjugate prior

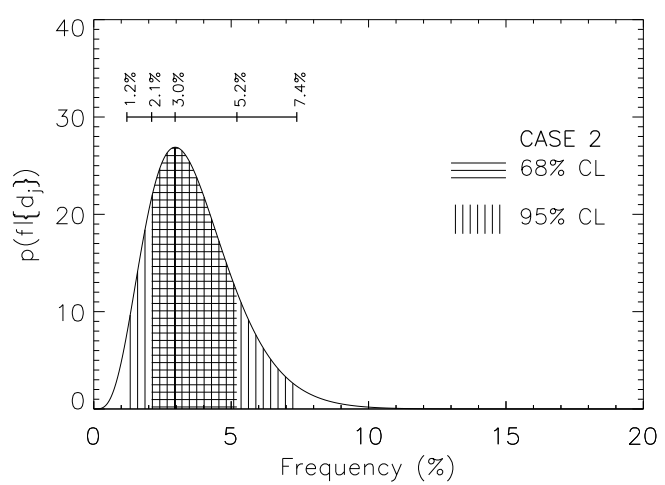

(b) $0.01<Q<0.05$ (case 2), conjugate prior

Fig. D.4. Companion frequency distribution for the three cases considered in the text derived from the merging of the LMS and the HMS samples.
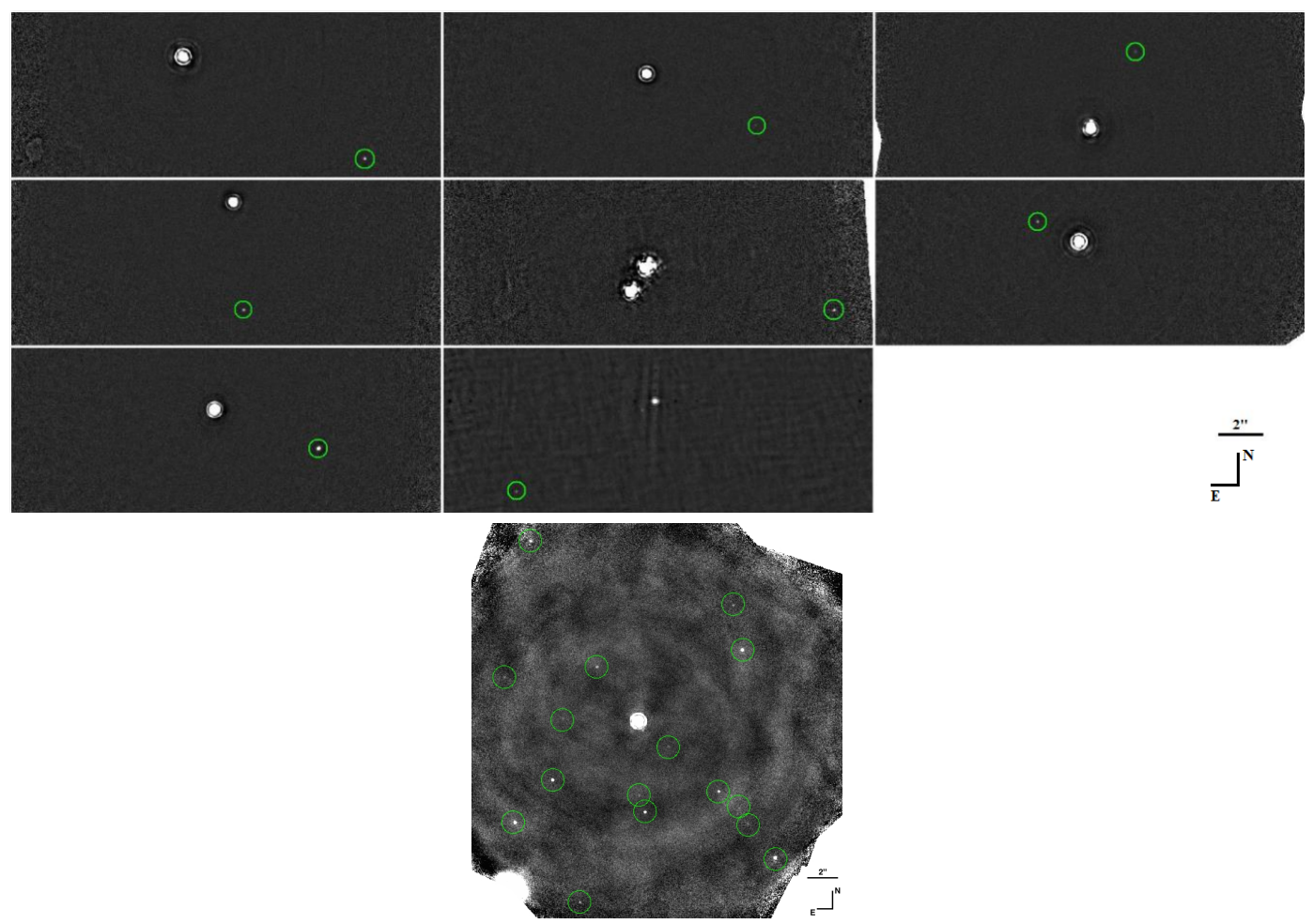

Fig. D.5. Background stars identified in the MASSIVE survey, highlighted with green circles. The primary M dwarfs are, from left to right and top to bottom: 2MASS J06255610-6003273, 2MASS J11210549-3845163, 2MASS J18465255-6210366, 2MASS J013655160647379, 2MASS J05015881+0958587, 2MASS J04522441-1649219, 2MASS J07285117-3015527, 2MASS J12073346-3932539, and 2MASS J15385757-5742273 (the image of this target is at the very bottom). Images are displayed using identical linear scalings. East is left and North is up. 
Table D.1. Close binary systems $\left(<1^{\prime \prime}\right)$ identified in our survey.

\begin{tabular}{cccc}
\hline \hline ID & $\begin{array}{c}\text { Approx. separation } \\
\left({ }^{\prime \prime}\right)\end{array}$ & $\begin{array}{c}\approx \Delta L^{\prime} \\
(\mathrm{mag})\end{array}$ & Ref. \\
\hline 2MASS J01033563-5515561 & 0.25 & 0.22 & \\
2MASS J01071194-1935359 & 0.45 & 0.09 & \\
2MASS J01231125-6921379 & $<0.07^{a}$ & & \\
2MASS J03363144-2619578 & $<0.12^{a}$ & & \\
2MASS J04373746-0229282 & 0.26 & 1.26 & Kasper et al. (2007) \\
2MASS J05015881+0958587 & 1.37 & 1.39 & Elliott et al. (2015) \\
2MASS J05195412-0723359 & 0.72 & 0.51 & \\
2MASS J05320450-0305291 & 0.18 & 0.63 & \\
2MASS J07285137-3014490 & $<0.12^{a}$ & & Daemgen et al. (2007) \\
2MASS J08173943-8243298 & 0.62 & 1.00 & \\
2MASS J13215631-1052098 & $<0.11^{a}$ & & \\
2MASS J14112131-2119503 & $<0.15^{a}$ & & \\
2MASS J19560294-3207186 & 0.20 & 1.04 & Elliott et al. (in prep.) \\
\hline
\end{tabular}

Notes. ${ }^{(a)}$ Stars' point spread function not resolved.

Table D.2. Estimated masses of our MASSIVE targets, using their absolute magnitudes and the BT-Settl models (Allard et al. 2012).

\begin{tabular}{cc}
\hline \hline ID & $\begin{array}{c}\text { Mass } \\
\left(M_{\text {Jup }}\right)\end{array}$ \\
\hline 2MASS J00243202-2522528 & 450 \\
2MASS J00251465-6130483 & 700 \\
2MASS J00255097-0957398 & 400 \\
2MASS J00452814-5137339 & 700 \\
2MASS J01033563-5515561 & 450 \\
2MASS J01231125-6921379 & 65 \\
2MASS J01365516-0647379 & 110 \\
2MASS J01521830-5950168 & 500 \\
2MASS J02224418-6022476 & 400 \\
2MASS J02365171-5203036 & 400 \\
2MASS J03350208+2342356 & 50 \\
2MASS J03363144-2619578 & 230 \\
2MASS J03472333-0158195 & 450 \\
2MASS J04141730-0906544 & 310 \\
2MASS J04373746-0229282 & 820 \\
2MASS J04433761+0002051 & 20 \\
2MASS J04464970-6034109 & 620 \\
2MASS J04522441-1649219 & 500 \\
2MASS J04533054-5551318 & 350 \\
2MASS J04593483+0147007 & 750 \\
2MASS J05004714-5715255 & 800 \\
2MASS J05064991-2135091 & 570 \\
2MASS J05195412-0723359 & 150 \\
2MASS J06085283-2753583 & $12 *$ \\
2MASS J06255610-6003273 & 570 \\
2MASS J07285117-3015527 & 250 \\
2MASS J08173943-8243298 & 650 \\
2MASS J10284580-2830374 & 90 \\
2MASS J10285555+0050275 & 450 \\
2MASS J11020983-3430355 & 250 \\
2MASS J11210549-3845163 & 570 \\
2MASS J11393382-3040002 & 150 \\
2MASS J11395113-3159214 & 15 \\
2MASS J12072738-3247002 & 500 \\
\hline
\end{tabular}

Notes. ${ }^{(*)}$ The association of this star (BPMG, Riedel et al. 2014; and Malo et al. 2013) were debated by Gagné et al. (2014b) who show that this target could be a member to the Columba association, but other radial velocity measurements are still needed to confirmed this. The mass of this object is quite low here, and is more certainly around 15-24 $M_{\text {Jup }}$, as reported by Riedel et al. (2014) and Gagné et al. (2014b). We however kept this low mass in order to have a consistent determination of all the stellar masses. 
Table D.2. continued.

\begin{tabular}{cc}
\hline \hline ID & $\begin{array}{c}\text { Mass } \\
\left(M_{\text {Jup }}\right)\end{array}$ \\
\hline 2MASS J12073346-3932539 & 20 \\
2MASS J12153072-3948426 & 700 \\
2MASS J12265135-3316124 & 350 \\
2MASS J13215631-1052098 & 182 \\
2MASS J15385757-5742273 & 175 \\
2MASS J16334161-0933116 & 600 \\
FS2003 0979 & 300 \\
2MASS J18465255-6210366 & 750 \\
2MASS J19560294-3207186 & 820 \\
2MASS J19560438-3207376 & 750 \\
2MASS J20333759-2556521 & 350 \\
2MASS J20450949-3120266 & 620 \\
2MASS J20450949-3120266 & 620 \\
2MASS J21100535-1919573 & 600 \\
2MASS J21443012-6058389 & 620 \\
2MASS J22445794-3315015 & 450 \\
2MASS J22450004-3315258 & 250 \\
2MASS J23301341-2023271 & 500 \\
2MASS J23323085-1215513 & 700 \\
2MASS J23381743-4131037 & 450 \\
\hline
\end{tabular}

Table D.3. Effective number of stars.

\begin{tabular}{cccc}
\hline \hline Survey & Case 1 & Case 2 & Case 3 \\
\hline MASSIVE & 45.9 & 40.0 & 23.2 \\
A-F survey & 27.6 & 28.3 & 23.9 \\
\hline
\end{tabular}

Table D.4. Detections of PMCs or brown dwarfs for MASSIVE and the A-F type star surveys, relative to our cases.

\begin{tabular}{|c|c|c|c|c|}
\hline System & Case 1 & Case 2 & Case 3 & Ref. \\
\hline $\begin{array}{l}\text { MASSIVE } \\
\text { 2MASS J01033563-5515561 } \\
\text { 2MASS J12073346-3932539 }\end{array}$ & $\begin{array}{l}\checkmark \\
\checkmark\end{array}$ & $\checkmark$ & & $\begin{array}{l}\text { Delorme et al. (2013) } \\
\text { Chauvin et al. (2004) }\end{array}$ \\
\hline $\begin{array}{c}\text { A-F survey } \\
\beta \text { Pictoris } \\
\text { HR } 8799 \\
\text { HIP } 95261\end{array}$ & $\begin{array}{l}\checkmark \\
\checkmark \\
\checkmark\end{array}$ & $\checkmark$ & $\begin{array}{l}\checkmark \\
\checkmark\end{array}$ & $\begin{array}{l}\text { Bonnefoy et al. (2014) } \\
\text { Pueyo et al. (2015) } \\
\text { Lowrance et al. (2000) }\end{array}$ \\
\hline
\end{tabular}

Notes. We selected the median values of the stellar and companion masses to estimate the case each system belongs to.

Table D.5. Comparison of the results from different surveys on M-dwarfs.

\begin{tabular}{cccc}
\hline \hline Survey & Planet frequency & Range considered & Our results within the same range \\
\hline $\mathrm{RV}+\mathrm{DI},(1)$ & $6.5 \pm 3 \%$ & {$[1-13] M_{\mathrm{Jup}},[0-20] \mathrm{AU}$} & {$[0-3.0] \%$} \\
micro lensing+RV, (2) & $3.8_{-2.0}^{+1.9} \%$ & {$[1-13] M_{\mathrm{Jup}}, 1-10^{5}$ day periods } & {$[0-2.0] \%$} \\
$\mathrm{RV},(3)$ & $<1 \%$ & $m \sin i=\left[10^{3}-10^{4}\right] M_{\text {Earth }}, 10^{3}-10^{4}$ day periods & {$[0-3.2] \%$} \\
\hline
\end{tabular}

References. (1) Montet et al. (2014); (2) Clanton \& Gaudi (2014); (3) Bonfils et al. (2013). 
Table D.6. Frequencies of PMC and SC with $68 \%$ confidence level, for different [mass, semi-major axis] ranges, using different priors, for specific detections.

\begin{tabular}{cccccc}
\hline \hline Frequency & Interval for 68\% CL & $\begin{array}{c}\text { Mass } \\
\left(M_{\text {Jup }}\right)\end{array}$ & $\begin{array}{c}\text { SMA } \\
(\mathrm{AU})\end{array}$ & Prior & Number of detections \\
\hline \multicolumn{7}{c}{ MASSIVE } \\
\hline $3.6 \%$ & {$[2.5,11.3] \%$} & {$[0.5,80]$} & {$[0.5,1000]$} & uniform & 1 \\
$3.6 \%$ & {$[2.4,8.1] \%$} & {$[0.5,80]$} & {$[0.5,1000]$} & natural conjugate & 1 \\
$2.3 \%$ & {$[1.6,7.3] \%$} & {$[2,14]$} & {$[8,400]$} & uniform & 1 \\
$2.3 \%$ & {$[1.6,8.1] \%$} & {$[2,14]$} & {$[8,400]$} & natural conjugate & 1 \\
$7.2 \%$ & {$[4.9,16.0] \%$} & {$[0.5,80]$} & {$[0.5,1000]$} & uniform & 2 \\
$7.2 \%$ & {$[5.1,12.6] \%$} & {$[0.5,80]$} & {$[0.5,1000]$} & natural conjugate & 2 \\
$4.4 \%$ & {$[3.0,9.6] \%$} & {$[2,14]$} & {$[8,400]$} & uniform & 2 \\
$4.4 \%$ & {$[3.1,7.6] \%$} & {$[2,14]$} & {$[8,400]$} & natural conjugate & 2 \\
\hline
\end{tabular}

Notes. The number of detections is represented in the last column: the number 1 is for the PMC detection around the target 2MASS0103(AB), the number 2 is for the PMCs detection around 2MASS0103(AB) and 2M1207b.

Table D.7. Kolmogorov-Smirnov statistics and associated probability for each case, and the probability of two populations based on a Monte Carlo simulation.

\begin{tabular}{lcc}
\hline \hline Mass ratio Q range & KS statistic & Probability of two populations \\
\hline Case $1, \forall Q$ & 0.71 & $74.2 \%$ \\
Case $2,0.01 \leq Q \leq 0.05$ & 0.23 & $20.6 \%$ \\
Case $3, Q<0.01$ & 0.82 & $74.5 \%$ \\
\hline
\end{tabular}

Table D.8. Observing conditions.

\begin{tabular}{ccccc}
\hline \hline Target & Airmass & $\begin{array}{c}\text { Parang. } \\
\left({ }^{\circ}\right)\end{array}$ & $\begin{array}{c}\text { Tau0 }(\mathrm{ms}) \\
(\mathrm{ms})\end{array}$ & Seeing \\
\hline 2MASS J00243202-2522528 & 1.0 & 20.9 & 1.1 & 1.20 \\
2MASS J00251465-6130483 & 1.3 & 27.0 & 1.5 & 0.94 \\
2MASS J00255097-0957398 & 1.0 & 44.2 & 1.9 & 0.99 \\
2MASS J00452814-5137339 & 1.1 & 30.8 & 1.0 & 1.30 \\
2MASS J01033563-5515561 & 1.2 & 8.4 & 2.4 & 0.69 \\
2MASS J01071194-1935359 & 1.0 & 69.9 & 2.2 & 1.00 \\
2MASS J01231125-6921379 & 1.4 & 10.4 & 2.1 & 0.81 \\
2MASS J01365516-0647379 & 1.1 & 30.4 & 1.6 & 1.13 \\
2MASS J01521830-5950168 & 1.2 & 32.5 & 1.3 & 1.01 \\
2MASS J02224418-6022476 & 1.2 & 10.8 & 1.8 & 1.08 \\
2MASS J02365171-5203036 & 1.1 & 25.9 & 2.7 & 1.34 \\
2MASS J03350208+2342356 & 1.5 & 2.0 & 2.8 & 0.60 \\
2MASS J03363144-2619578 & 1.0 & 9.0 & 2.8 & 0.64 \\
2MASS J03472333-0158195 & 1.1 & 10.5 & 2.2 & 0.84 \\
2MASS J04141730-0906544 & 1. & 5.2 & 2.4 & 0.72 \\
2MASS J04373746-0229282 & 1.1 & 30.6 & 4.7 & 1.06 \\
2MASS J04433761+0002051 & 1.1 & 17.5 & 8.9 & 0.83 \\
2MASS J04464970-6034109 & 1.3 & 33.0 & 3.8 & 0.93 \\
2MASS J04522441-1649219 & 1.0 & 56.3 & 2.8 & 0.65 \\
2MASS J04533054-5551318 & 1.2 & 22.1 & 1.4 & 0.98 \\
2MASS J04593483+0147007 & 1.2 & 0.0 & 5.0 & 0.94 \\
2MASS J05004714-5715255 & 1.2 & 14.7 & 3.8 & 0.87 \\
2MASS J05015881+0958587 & 1.2 & 8.9 & 2.3 & 0.76 \\
2MASS J05064991-2135091 & 1.2 & 0.0 & 2.8 & 0.94 \\
2MASS J05195412-0723359 & 1.1 & 5.7 & 5.5 & 1.16 \\
2MASS J05320450-0305291 & 1.1 & 33.2 & 2.1 & 0.84 \\
2MASS J06085283-2753583 & 1.0 & 40.2 & 2.1 & 0.88 \\
2MASS J06255610-6003273 & 1.2 & 16.9 & 7.0 & 0.77 \\
2MASS J07285117-3015527 & 1.0 & 65.8 & 2.6 & 0.70 \\
2MASS J07285137-3014490 & 1.0 & 58.1 & 1.4 & 1.27 \\
\hline
\end{tabular}


Table D.8. continued.

\begin{tabular}{ccccc}
\hline \hline Target & Airmass & $\begin{array}{c}\text { Parang. } \\
\left({ }^{\circ}\right)\end{array}$ & $\begin{array}{c}\text { Tau0 }(\mathrm{ms}) \\
(\mathrm{ms})\end{array}$ & Seeing \\
\hline 2MASS J08173943-8243298 & 1.9 & 2.0 & 5.5 & 0.91 \\
2MASS J10284580-2830374 & 1.0 & 76.5 & 7.5 & 1.07 \\
2MASS J10285555+0050275 & 1.1 & 15.5 & 2.9 & 0.94 \\
2MASS J11020983-3430355 & 1.0 & 43.0 & 1.8 & 1.18 \\
2MASS J11210549-3845163 & 1.0 & 34.7 & 9.6 & 0.89 \\
2MASS J11393382-3040002 & 1.0 & 39.7 & 2.0 & 1.02 \\
2MASS J11395113-3159214 & 1.1 & 17.5 & 8.9 & 0.83 \\
2MASS J12072738-3247002 & 1.0 & 67.9 & 21.5 & 0.83 \\
2MASS J12073346-3932539 & 1.1 & 15.5 & 2.9 & 0.94 \\
2MASS J12153072-3948426 & 1.2 & 5.0 & 4.7 & 0.58 \\
2MASS J12265135-3316124 & 1.0 & 19.5 & 10.2 & 0.82 \\
2MASS J13215631-1052098 & 1.0 & 28.8 & 9.2 & 0.88 \\
2MASS J14112131-2119503 & 1.0 & 54.9 & 6.9 & 1.13 \\
2MASS J15385757-5742273 & 1.3 & 30.0 & 22.5 & 0.67 \\
2MASS J16334161-0933116 & 1.1 & 35.6 & 1.5 & 0.99 \\
FS2003 0979 & 1.0 & 25.9 & 1.5 & 0.95 \\
2MASS J18465255-6210366 & 1.3 & 18.7 & 1.3 & 1.13 \\
2MASS J19560294-3207186 & 1.0 & 58.4 & 1.1 & 1.33 \\
2MASS J19560438-3207376 & 1.0 & 65.6 & 4.0 & 0.67 \\
2MASS J20333759-2556521 & 1.0 & 27.2 & 1.3 & 1.15 \\
2MASS J20450949-3120266 & 1.0 & 54.4 & 3.3 & 0.54 \\
2MASS J21100535-1919573 & 1.0 & 55.8 & 1.4 & 0.93 \\
2MASS J21443012-6058389 & 1.3 & 24.8 & 1.1 & 1.20 \\
2MASS J22445794-3315015 & 1.2 & 0.0 & 5.0 & 0.94 \\
2MASS J22450004-3315258 & 1.2 & 0.0 & 5.0 & 0.94 \\
2MASS J23301341-2023271 & 1.0 & 54.3 & 0.9 & 2.10 \\
2MASS J23323085-1215513 & 1.5 & 0.0 & 4.9 & 1.33 \\
2MASS J23381743-4131037 & 1.1 & 38.6 & 2.5 & 0.65 \\
\hline
\end{tabular}

Table D.9. $5 \sigma$ median contrast achieved within and beyond $0.5^{\prime}$, and boundaries between the inner and outer regions for stars reduced with ADI.

\begin{tabular}{cccc}
\hline \hline Target & $\leq 0.5^{\prime}$ & $>0.5^{\prime}$ & $\begin{array}{c}\text { Boundary } \\
\text { (as) }\end{array}$ \\
\hline 2MASS J00243202-2522528 & $6.38 \pm 0.06$ & $8.34 \pm 0.01$ & 0.41 \\
2MASS J00251465-6130483 & $8.02 \pm 0.06$ & $10.04 \pm 0.01$ & 0.62 \\
2MASS J00255097-0957398 & $7.13 \pm 0.03$ & $8.14 \pm 0.01$ & 0.51 \\
2MASS J00452814-5137339 & $7.66 \pm 0.08$ & $9.52 \pm 0.01$ & 0.79 \\
2MASS J01033563-5515561 & $5.38 \pm 0.19$ & $7.91 \pm 0.01$ & \\
2MASS J01071194-1935359 & $7.35 \pm 0.19$ & $10.03 \pm 0.02$ & 0.62 \\
2MASS J01231125-6921379 & $6.08 \pm 0.06$ & $6.32 \pm 0.01$ & \\
2MASS J01365516-0647379 & $7.95 \pm 0.06$ & $8.84 \pm 0.01$ & 0.65 \\
2MASS J01521830-5950168 & $8.18 \pm 0.04$ & $9.32 \pm 0.01$ & 0.73 \\
2MASS J02224418-6022476 & $6.52 \pm 0.10$ & $9.12 \pm 0.01$ & \\
2MASS J02365171-5203036 & $8.39 \pm 0.06$ & $9.84 \pm 0.01$ & 0.79 \\
2MASS J03350208+2342356 & $4.33 \pm 0.08$ & $4.58 \pm 0.01$ & \\
2MASS J03363144-2619578 & $6.74 \pm 0.08$ & $7.69 \pm 0.01$ & \\
2MASS J03472333-0158195 & $7.74 \pm 0.06$ & $10.19 \pm 0.02$ & 0.60 \\
2MASS J04141730-0906544 & $6.64 \pm 0.09$ & $7.54 \pm 0.01$ & \\
2MASS J04373746-0229282 & $6.07 \pm 0.29$ & $11.29 \pm 0.02$ & 0.38 \\
2MASS J04433761+0002051 & $6.08 \pm 0.04$ & $6.81 \pm 0.01$ & 0.38 \\
2MASS J04464970-6034109 & $8.44 \pm 0.08$ & $9.94 \pm 0.01$ & 0.79 \\
2MASS J04522441-1649219 & $9.03 \pm 0.09$ & $10.90 \pm 0.01$ & 0.95 \\
2MASS J04533054-5551318 & $6.10 \pm 0.09$ & $9.69 \pm 0.03$ & 1.00 \\
2MASS J04593483+0147007 & $6.93 \pm 0.13$ & $10.54 \pm 0.02$ & \\
2MASS J05004714-5715255 & $8.18 \pm 0.06$ & $10.74 \pm 0.02$ & 0.68 \\
2MASS J05015881+0958587 & $7.73 \pm 0.12$ & $11.65 \pm 0.02$ & \\
2MASS J05064991-2135091 & $7.67 \pm 0.11$ & $10.83 \pm 0.02$ & \\
\hline
\end{tabular}


Table D.9. continued.

\begin{tabular}{cccc}
\hline \hline Target & $\leq 0.5^{\prime}$ & $>0.5^{\prime}$ & $\begin{array}{c}\text { Boundary } \\
\text { (as) }\end{array}$ \\
\hline 2MASS J05195412-0723359 & $6.30 \pm 0.08$ & $6.77 \pm 0.01$ & \\
2MASS J05320450-0305291 & $8.03 \pm 0.48$ & $10.64 \pm 0.01$ & 0.84 \\
2MASS J06085283-2753583 & $5.18 \pm 0.06$ & $5.53 \pm 0.01$ & \\
2MASS J06255610-6003273 & $8.56 \pm 0.08$ & $11.93 \pm 0.02$ & 0.57 \\
2MASS J07285117-3015527 & $8.11 \pm 0.06$ & $9.66 \pm 0.01$ & 0.60 \\
2MASS J07285137-3014490 & $7.66 \pm 0.08$ & $11.18 \pm 0.02$ & 1.06 \\
2MASS J08173943-8243298 & $7.21 \pm 0.12$ & $10.67 \pm 0.02$ & \\
2MASS J10284580-2830374 & $7.04 \pm 0.04$ & $8.03 \pm 0.01$ & 0.46 \\
2MASS J10285555+0050275 & $8.78 \pm 0.06$ & $11.80 \pm 0.02$ & 1.14 \\
2MASS J11020983-3430355 & $5.52 \pm 0.03$ & $5.94 \pm 0.01$ & 0.35 \\
2MASS J11210549-3845163 & $8.64 \pm 0.05$ & $9.66 \pm 0.01$ & 0.70 \\
2MASS J11393382-3040002 & $7.35 \pm 0.06$ & $8.70 \pm 0.01$ & 0.57 \\
2MASS J1395113-3159214 & $5.98 \pm 0.08$ & $6.49 \pm 0.01$ & \\
2MASS J12072738-3247002 & $8.24 \pm 0.07$ & $10.02 \pm 0.01$ & 0.70 \\
2MASS J12073346-3932539 & $5.32 \pm 0.08$ & $6.14 \pm 0.03$ & \\
2MASS J12153072-3948426 & $6.48 \pm 0.09$ & $9.43 \pm 0.02$ & \\
2MASS J12265135-3316124 & $6.59 \pm 0.05$ & $7.63 \pm 0.01$ & 0.41 \\
2MASS J13215631-1052098 & $7.87 \pm 0.09$ & $9.06 \pm 0.01$ & 0.68 \\
2MASS J14112131-2119503 & $6.04 \pm 0.07$ & $6.54 \pm 0.01$ & 0.33 \\
2MASS J15385757-5742273 & $7.50 \pm 0.04$ & $10.44 \pm 0.02$ & 0.60 \\
2MASS J16334161-0933116 & $7.89 \pm 0.06$ & $9.77 \pm 0.01$ & 0.62 \\
FS2003 0979 & $7.71 \pm 0.06$ & $9.04 \pm 0.02$ & 0.57 \\
2MASS J18465255-6210366 & $8.39 \pm 0.04$ & $9.36 \pm 0.01$ & 1.00 \\
2MASS J19560294-3207186 & $6.78 \pm 0.42$ & $9.49 \pm 0.01$ & 0.60 \\
2MASS J19560438-3207376 & $8.61 \pm 0.06$ & $9.61 \pm 0.01$ & 0.70 \\
2MASS J20333759-2556521 & $6.14 \pm 0.07$ & $7.94 \pm 0.01$ & 0.57 \\
2MASS J20450949-3120266 & $9.40 \pm 0.08$ & $12.41 \pm 0.02$ & 1.06 \\
2MASS J21100535-1919573 & $7.55 \pm 0.09$ & $10.01 \pm 0.02$ & 0.60 \\
2MASS J21443012-6058389 & $8.58 \pm 0.06$ & $9.63 \pm 0.01$ & 0.70 \\
2MASS J22445794-3315015 & $7.07 \pm 0.10$ & $10.37 \pm 0.02$ & \\
2MASS J22450004-3315258 & $6.29 \pm 0.13$ & $8.96 \pm 0.01$ & \\
2MASS J23301341-2023271 & $7.50 \pm 0.08$ & $10.44 \pm 0.02$ & 0.62 \\
2MASS J23323085-1215513 & $6.94 \pm 0.12$ & $10.02 \pm 0.02$ & \\
2MASS J23381743-4131037 & $8.01 \pm 0.09$ & $11.63 \pm 0.01$ & 0.38 \\
\hline & & & \\
\hline
\end{tabular}

